\title{
Determination of the cross sections for the production of fragments from relativistic nucleus-nucleus interactions. I. Measurements
}

\author{
J. R. Cummings, ${ }^{(1), *}$ W. R. Binns, ${ }^{(2)}$ T. L. Garrard, ${ }^{(3)}$ M. H. Israel, ${ }^{(2)}$ J. Klarmann, ${ }^{(2)}$ E. C. Stone, ${ }^{(3)}$ and \\ C. J. Waddington ${ }^{(1)}$ \\ ${ }^{11}$ School of Physics and Astronomy, University of Minnesota, Minneapolis, Minnesota 55455 \\ ${ }^{(2)}$ Department of Physics and the McDonnell Center for the Space Sciences, Washington University, St. Louis, Missouri 63130 \\ ${ }^{(3)}$ George W. Downs Laboratory, California Institute of Technology, Pasadena, California 91125
}

(Received 21 September 1989; revised manuscript received 3 May 1990)

\begin{abstract}
Relativistic iron, lanthanum, holmium, and gold projectile nuclei with several different energies have been fragmented in targets of polyethylene, carbon, aluminum, copper, and lead. Our detectors cleanly resolve the individual charges of the heaviest of these fragments and provide some limited information on the masses. We have measured 1256 elemental partial cross sections for the production of fragments from interactions in these target materials. Values have been derived for another 417 cross sections in a hydrogen medium. These cross sections depend on the energy and mass of the projectile nuclei as well as on the nature of the target. Total charge-changing cross sections were also found, but only in a composite target, and have been shown to be weakly dependent on energy. The mean mass losses observed for fragments that have lost a few protons show that typically many neutrons are lost with each proton, producing fragment nuclei that must be highly proton rich, and consequently very unstable. The cross sections for charge pickup on heavy targets show a rapid increase with decreasing energy, particularly for the heaviest targets. The systematics of the dependencies of the partial cross sections will be discussed in a companion paper.
\end{abstract}

\section{INTRODUCTION}

In this paper we report the experimental results from our analysis of heavy-fragment nuclei produced by interactions of several different projectiles in various target materials observed in an inclusive experiment conducted at the Lawrence Berkeley Laboratory's (LBL) Bevalac particle accelerator. In a companion paper $^{1}$ we will present global fits to the data, based on the systematics observed in the trends of the cross sections for the production of fragments from these projectiles. These fits allow the prediction of those cross sections that are not measured directly.

The experimental objective of this experiment was to measure the elemental cross sections for the production of heavy-fragment nuclei from charge-changing interactions between heavy-projectile nuclei with energies of at least several hundred $\mathrm{MeV} /$ nucleon and various target nuclei. Our scientific motivation was predominantly directed at the astrophysical problems of the propagation of cosmic ray nuclei through the interstellar medium, and at the nuclear physics of these peripheral relativistic nucleus-nucleus interactions. In addition, we were also concerned with the application of the cross sections and other results to the calibration and correction of data from instruments used to detect cosmic ray nuclei in the vicinity of the Earth. In particular we were concerned with how they related to our own earlier experiment ${ }^{2}$ on the NASA High-Energy Astronomy Observatory (HEAO 3) Heavy-Nuclei Experiment (HNE), which was designed to detect the very rare ultraheavy (UH) nuclei, those with $Z \geq 30$, present in the cosmic radiation.
These interactions of high-energy heavy nuclei with various target nuclei are interesting in themselves, as an aspect of nuclear physics still not thoroughly understood. $^{3}$ Extensive earlier studies of fragmentation in peripheral interactions have been mainly confined to studies with projectile nuclei lighter than nickel and hence with smaller total kinetic energies. ${ }^{4-7}$ The systematics of the yields of the different fragment species that we find in this work appear to have no obvious theoretical justification, but are so striking and simple that they must surely represent a fundamental aspect of the manner in which heavy nuclei are disassembled during the peripheral interactions which leave the large fragments that we are studying in this work. ${ }^{8,9}$ With our detector we could only observe the summed charge of the particles produced by the breakup of the projectile nucleus and hence we were unable to study the multifragmentation that is known to occur with greater frequency as the interactions become more central. ${ }^{10,11}$ However, because of the dependence of the detector signals on $Z^{2}$, in interactions where the projectile is less completely disrupted, the heaviest fragment dominates the total signal, allowing an inclusive measurement of the yields of these fragments.

We have conducted two previous experiments ${ }^{12,13}$ at the LBL Bevalac along similar lines to the one reported in this paper. The detectors have undergone significant improvements, with better resolution and signal-to-noise ratios. At the same time the capability of the Bevalac to provide a wide range of projectiles, with different energies and species has also improved. The experiment of Ref. 12 sampled one projectile $(\mathrm{Au})$ at one energy on two tar- 
gets and could be characterized by a charge resolution for energetic gold nuclei of $\approx 0.4$ charge units $(\mathrm{cu})$. The experiment of Ref. 13 sampled four projectiles on at most three targets at one energy each, with a charge resolution of $\approx 0.3 \mathrm{cu}$. The present experiment sampled four projectiles, each with up to six energies, mostly on four targets, and with a charge resolution of $\approx 0.2 \mathrm{cu}$.

Significant results from the analysis of the earlier experiments to which we will need to refer to this paper included the derivation of an expression for the total charge-changing cross sections $\Sigma$ of heavy-projectile nuclei of mass number $A_{P}$ on various targets of mass number $A_{T}$. The expression derived ${ }^{14}$ is

$$
\Sigma=10 \pi r_{0}^{2}\left[A_{P}^{1 / 3}+A_{T}^{1 / 3}-0.209\left(A_{P}+A_{T}\right)^{1 / 3}\right]^{2},
$$

where $r_{0}$ is 1.35 and $\Sigma$ is in mb. This formula fits the cross sections for projectile nuclei heavier than iron. Earlier, Westfall et al. ${ }^{15}$ derived a similar expression, but with a fixed overlap term, based on the original formula of Bradt and Peters, ${ }^{16}$ for projectile nuclei lighter than iron, of the form:

$$
\Sigma_{W}=10 \pi r_{0}^{2}\left(A_{P}^{1 / 3}+A_{T}^{1 / 3}-0.83\right)^{2} .
$$

For both formulas, one must substitute 0.089 for the mass number of 1 when a hydrogen target is considered. These two expressions match very closely for ironprojectile nuclei $A_{P}=56$ but diverge outside their ranges of applicability. In both cases it is assumed that there is no significant energy dependence over the range of energies studies. In Sec. III E we show that this assumption is only valid to some $10 \%$, but that this weak energy dependence only introduces a $1 \%$ systematic uncertainty into our derived partial cross sections.

In this study we have mainly expressed our results in terms of the kinetic energy per nucleon of the projectile nuclei, rather than in terms of the total kinetic energy of the projectile, in either frame of reference. The energy per nucleon represents the velocity of the nuclei and experimentally describes the expected response from our detectors. Furthermore, from an astrophysical viewpoint, it describes and organizes the nuclei observed in the cosmic radiation. Earlier studies of target fragmentation, ${ }^{3-7,17}$ done mainly with lighter projectiles, have generally supported the hypothesis of "limiting fragmentation" where the total projectile kinetic energy is the important parameter that describes the fragmentation in peripherical interactions, with the yields reaching asymptotic values at a few $\mathrm{GeV}$. In our case, where the projectiles were almost always significantly heavier than the target nuclei, the relevant energy would be that of the target nucleus as seen in the rest frame of the projectile. In the analysis of our data, presented in the companion paper, we have examined this hypothesis and found that for these heavy projectiles and light targets, limiting fragmentation is only accurate to some $30 \%$. Hence, while assuming its validity can allow us to make some useful predictions about the expected behavior of heavy highenergy projectiles on a hydrogen target, it provides a less precise representation of the fragmentation on heavy tar- gets than that obtained from our own representations, which are expressed in terms of the energy per nucleon, and appear to have an accuracy of better than $10 \%$.

\section{EXPERIMENTAL}

\section{A. Description of counters}

The detector for the run reported here consisted of an array of parallel-plate gas-ionization counters and light diffusion box Cherenkov counters with Pilot 425 radiators. A multiwire proportional counter (MWPC) was mounted directly in front of the Cherenkov counters and was used for position determination. This detector is illustrated schematically in Fig. 1, which shows the configuration of counters and the location of the target holder. Projectile nuclei entered I-1 after traversing a thin vacuum window and some $2.5 \mathrm{~m}$ of air. The thicknesses of all the materials used in the detector, apart from the targets, are listed in Table I.

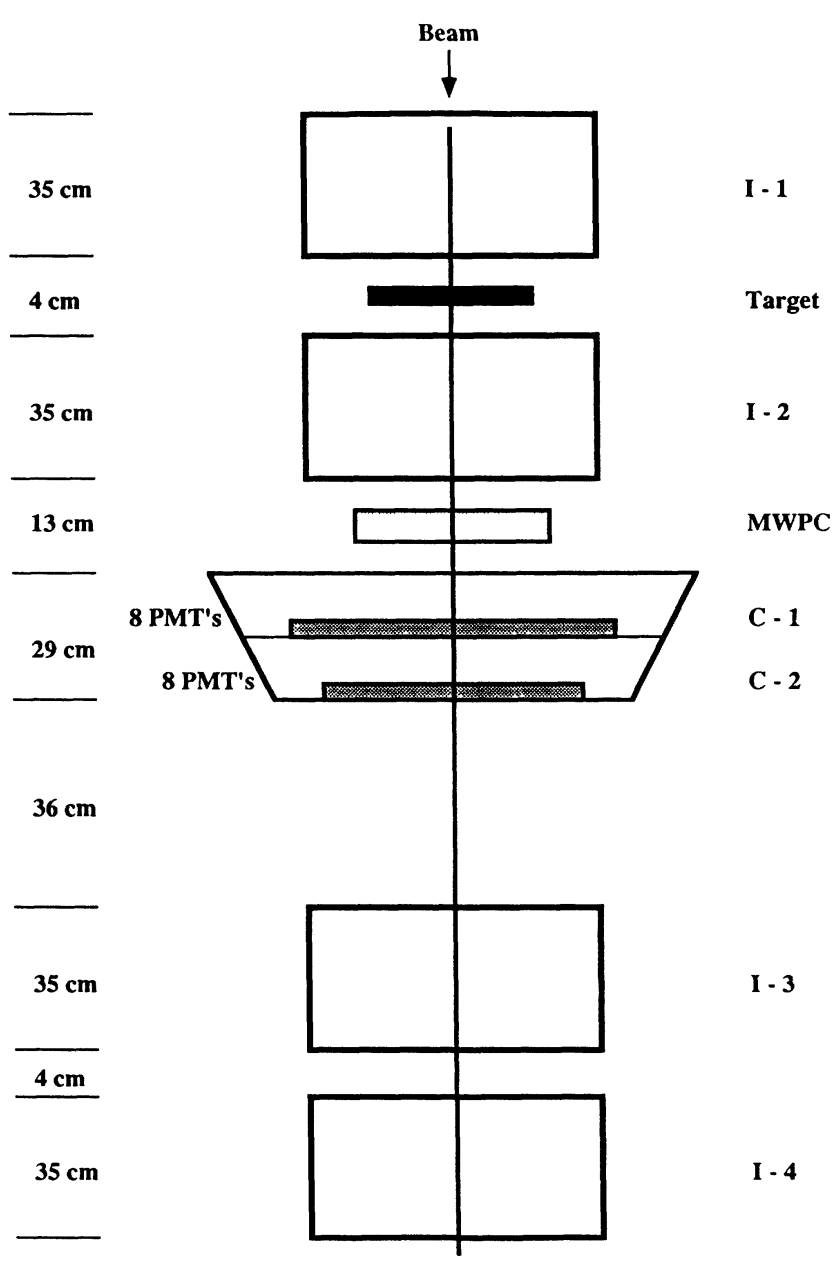

FIG. 1. Schematic diagram of the detector. Ionization counters are designated I and Cherenkov counters C. The target and the multiwire proportional counters, MWPC, are mounted as shown. 
TABLE I. Thickness of all permanent materials used in the detector.

\begin{tabular}{lcccr}
\hline \hline \multicolumn{1}{c}{ Name } & \multicolumn{3}{c}{ Ion chamber } & \\
\hline em shield & Material & $\mathrm{cm}$ & $\mathrm{g} / \mathrm{cm}^{2}$ & $\%$ \\
Space & aluminum & 0.0127 & 0.0344 & 25.1 \\
Bag & air & 2.625 & 0.00339 & 2.5 \\
Gas & Mylar & 0.00127 & 0.0017 & 1.2 \\
Electrode & P10 & 1.00 & 0.01067 & 1.2 \\
Gas & Mylar & 0.000635 & 0.000826 & 0.6 \\
Electrode & P10 & 7.015 & 0.0126 & 9.2 \\
Gas & Mylar & 0.000635 & 0.000826 & 0.6 \\
Electrode & P10 & 7.015 & 0.0126 & 9.2 \\
Gas & Mylar & 0.000635 & 0.000826 & 0.6 \\
Electrode & P10 & 7.015 & 0.0126 & 9.2 \\
Gas & Mylar & 0.000635 & 0.000826 & 0.6 \\
Electrode & P10 & 7.015 & 0.0126 & 9.2 \\
Gas & Mylar & 0.000635 & 0.000826 & 0.6 \\
Bag & P10 & 1.00 & 0.00167 & 1.2 \\
Space & Mylar & 0.00127 & 0.0017 & 1.2 \\
em shield & air & 2.625 & 0.00339 & 2.5 \\
& aluminum & 0.0127 & 0.0344 & 25.1
\end{tabular}

\begin{tabular}{lcccr}
\multicolumn{1}{c}{ Name } & Material & $\begin{array}{c}\text { Cherenkov detector } \\
\mathrm{cm}\end{array}$ & $\mathrm{g} / \mathrm{cm}^{2}$ & $\%$ \\
\hline Light window & aluminum & 0.0127 & 0.0343 & 2.2 \\
Paint & "aluminum" & & 0.0055 & 0.4 \\
Millipore & polyethylene & & 0.0062 & 0.4 \\
Space & air & 14.4 & 0.0186 & 1.2 \\
Radiator & Lucite & 0.569 & 0.677 & 44.0 \\
Millipore & polyethylene & & 0.0062 & 0.4 \\
Paint & "aluminum" & & 0.006 & 0.4 \\
Light window & aluminum & 0.0127 & 0.0343 & 2.2 \\
Paint & "aluminum" & & 0.0055 & 0.4 \\
Millipore & polyethylene & & 0.0062 & 0.4 \\
Space & air & 13.5 & 0.0174 & 1.1 \\
Radiator & Lucite & 0.566 & 0.674 & 43.8 \\
Millipore & polyethylene & & 0.0062 & 0.4 \\
Paint & "aluminum" & & 0.006 & 0.4 \\
Light window & aluminum & 0.0127 & 0.0343 & 2.2
\end{tabular}

\begin{tabular}{|c|c|c|c|c|}
\hline \multicolumn{5}{|c|}{ MWPC } \\
\hline Name & Material & $\mathrm{cm}$ & $\mathrm{g} / \mathrm{cm}^{2}$ & $\%$ \\
\hline Window & Mylar & 0.000635 & 0.000826 & 2.6 \\
\hline Gas & $\mathrm{Ar}-5 \% \quad \mathrm{CO}_{2}$ & 0.1778 & 0.000329 & 1.0 \\
\hline Ground plane & Mylar & 0.000635 & 0.000826 & 2.6 \\
\hline Gas & $\mathrm{Ar}-5 \% \quad \mathrm{CO}_{2}$ & 0.1176 & 0.002055 & 6.4 \\
\hline Cathode & Mylar & 0.00127 & 0.001651 & 5.1 \\
\hline Gas & $\mathrm{Ar}-5 \% \quad \mathrm{CO}_{2}$ & 0.6985 & 0.001286 & 4.0 \\
\hline Ground plane & Mylar & 0.000635 & 0.000826 & 2.6 \\
\hline Gas & $\mathrm{Ar}-5 \% \quad \mathrm{CO}_{2}$ & 0.70358 & 0.006344 & 19.6 \\
\hline Cathode & Mylar & 0.00127 & 0.001651 & 5.1 \\
\hline Gas & $\mathrm{Ar}-5 \% \quad \mathrm{CO}_{2}$ & 1.1176 & 0.002055 & 6.4 \\
\hline Ground plane & Mylar & 0.000635 & 0.000826 & 2.6 \\
\hline Gas & $\mathrm{Ar}-5 \% \quad \mathrm{CO}_{2}$ & 1.1778 & 0.000329 & 1.0 \\
\hline Window & Mylar & 0.000635 & 0.000826 & 2.6 \\
\hline Gas & $\mathrm{Ar}-5 \% \quad \mathrm{CO}_{2}$ & 0.49276 & 0.000906 & 2.8 \\
\hline Space & air & 9 & 0.01161 & 35.9 \\
\hline
\end{tabular}


TABLE I. (Continued).

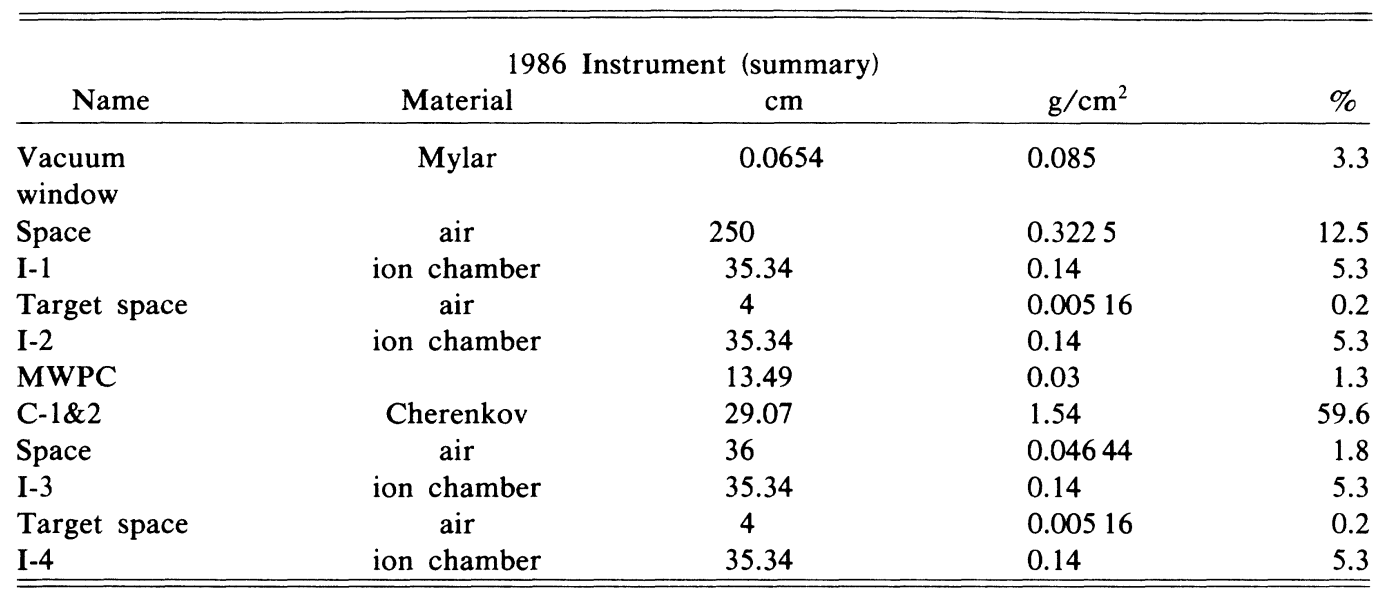

Ion counters yield signals proportional to the ionization produced by fast-moving charged particles in the gas. For particles with a unique velocity the signal should be proportional to the square of the charge, and no evidence of saturation effects was seen in these counters. ${ }^{18}$ The electrodes in the ionization counters were made of thin foils (aluminized Mylar) and hence the thickness of each counter was only about 0.002 of a nuclear interaction length for UH nuclei. The resolution of these ion counters showed an excellent improvement over those used in earlier runs, due to greater electrical stability and an increased thickness of gas resulting in greater statistical accuracy. Each counter consisted of four 7cm-wide electrode gaps or chambers filled with P10 gas, a mixture of argon with $10 \%$ methane.

The Cherenkov counters were two structurally connected but luminously separate diffusion boxes, basically truncated square pyramidal aluminum boxes, coated with white paint. The phototubes were angled away from the radiators, so that light received at the tubes was reflected at least once from the surface of the boxes. The paint served to diffuse the light from the radiator so as to increase the uniformity of the signal over the area of the detector. The radiators were each $0.57-\mathrm{cm}$-thick Pilot 425 plastic, which is chemically similar to lucite $\left(\mathrm{C}_{5} \mathrm{H}_{8} \mathrm{O}_{2}\right)$, but doped with a wave shifter which converts ultraviolet light to blue and reemits it isotropically. This wave shifter approximately doubles the signal detectable by the phototubes. Each radiator was $\approx 0.1$ of an interaction length thick for UH nuclei. Each counter had eight phototubes arranged in a plane perpendicular to the beam axis, two on each side.

The resolution of both the Cherenkov counters and the ion chambers was improved over that obtained previously. The resolution from the data reported in Ref. 13 for xenon projectiles with an energy of $1239 \mathrm{MeV} /$ nucleon in the center of the target was about $0.4 \mathrm{cu}$ for the ion chambers and $0.17 \mathrm{cu}$ for the Cherenkov counter. ${ }^{13}$ In the data reported here, the comparable values were 0.31 and $0.12 \mathrm{cu}$, respectively, for lanthanum projectiles with an energy of $1161 \mathrm{MeV} /$ nucleon in the center of the target. The improved resolution of the ion chambers allowed us to exclude events in which the projectile nucleus (or fragment nucleus produced in a target) interacted in the material of the Cherenkov detector. In the worst case, that of the gold projectiles, interacting nuclei that produced fragments with a charge change, $\Delta Z$, of $|\Delta Z| \leq 2 \mathrm{cu}$, were not completely eliminated, and, in addition, there were a few interactions in the small amount of material in the ion chambers. Hence we used runs with no target present, target-out runs, at corresponding energies to the target-in runs to correct for such interactions. This correction is described in Sec. III C 1.

\section{B. Corrections to raw data}

Each event was defined by 16 pulse-height signals, one from each detector, and bits which indicated the status of the detectors (triggered or not triggered). Not all particles passing through the detector triggered a recordable event, and not all recorded events had acceptable signals (Sec. II B 1). Nor were all recorded events initiated by particles of the projectile type (Sec. II B 3). In addition, the signals from the Cherenkov detectors had to be corrected for a systematic variation with the position of the particle path in the radiators, even though this variation was much less than that in the 1984 experiment (Sec. II B 2). In the following subsections we discuss a number of these corrections.

\section{Number of incident particles}

We chose triggers in the multiwire proportional counter as the criteria for an event to be recorded during the run. The reasons were mostly historical. Because it was the least sensitive detector, triggers in all four of the MWPC's signal channels would guarantee measurable signals in the other detectors. It was also necessary to have a reliable determination of the trajectory of every particle through the Cherenkov radiators, since the signal in the Cherenkov counter varied significantly with position. This led to the MWPC being placed behind the target, close to the Cherenkov counter. As a consequence particles undergoing collisions in the target which caused them to break up into small fragments (with the largest fragment being less than or approximately $0.33 Z_{\text {proj }}$, and 
the rest of the mass in singly or doubly charged particles) did not trigger the system and hence were not recorded. We estimated the number of unrecorded legitimate projectile particles to vary between $5 \%$ and $30 \%$ of the recorded particles, depending on the setting of the discriminator levels and the type and energy of the projectile. Thus the total numbers of projectile particles entering the detector are not measured and we can only determine relative, but not absolute, total or partial interaction cross sections directly from the data.

However, the number of incident projectile particles can be deduced from a knowledge of the total cross sections, the thickness of the material in the detector, and the number of surviving projectile particles. Hence, by using Eq. (1) or (2), as appropriate, absolute cross sections can be determined. These absolute cross sections are not very sensitive to the precise values assumed for the total cross sections, since none of the targets was more than 0.25 of a mean free path thick. In addition, we can perform a check on Eq. (1) by using the material of the Cherenkov detector as a target. These data provide us with some indications of the possible energy dependence or lack of it for total charge-changing cross sections. Details of the analysis of the total cross sections are given in Sec. III E.

\section{Mapping correction and position selection}

In previous runs the mapping correction for the nonuniformity of response over the area of the Cherenkov radiator was found to be as much as $\pm 7 \%$, which for Au nuclei implied the need to apply a correction of up to $3 \mathrm{cu}$. For this run, the Cherenkov detector was much improved, and the maximum mapping correction was about $\pm 1.5 \%$, or about $0.6 \mathrm{cu}$ for $\mathrm{Au}$ nuclei. There were no significant mapping corrections for the ion chambers in any of the runs.

In order to avoid any saturation effects in the ion chambers the projectiles from the Bevalac were defocused over a spot of several $\mathrm{cm}$ in diameter. We measured the positions of the particles in our detector with the MWPC, with an accuracy of $1 \mathrm{~mm}$. It was assumed that the positions in the MWPC could be mapped directly on the radiators, neglecting any divergence of the projectile or the produced fragments. Even at the lowest energies used here, where fragments could be emitted at angles of up to 35 mrad from the projectile direction, ${ }^{19}$ this divergence could introduce an uncertainty of less than $1.4 \mathrm{~cm}$ in the location of the impact point in the second radiator. This location uncertainty would lead to a charge uncertainty of less than $0.1 \mathrm{cu}$. At higher energies, where the Cherenkov signal is more important to the overall charge resolution, these uncertainties would be appreciably less, because the angular spread of the fragments is reduced.

Using intentionally defocused beams of $\mathrm{La}$ and $\mathrm{Fe}$ projectiles, we constructed signal maps of the two Cherenkov radiators over the central part of the radiator. The two sets of maps thus constructed did not differ significantly. In this analysis, the $\mathrm{La}$ maps were used, because $\mathrm{La}$ is closer in charge and signal level to most of our data. These maps were used to correct the Cherenkov signals.
Over the range of positions used, the maximum correction was less than $1.6 \%$, with an average correction of $0.4 \%$.

\section{Selection of projectile particles}

The beam from the Bevalac was not completely "clean" but contained a small fraction $(\leq 2 \%)$ of particles with different charge than those injected, presumably produced by interactions earlier in the beam path. In addition, there were some pile-up events with two or more particles entering the detector within the coincidence window and having signals double or triple the signals of single projectile particles. We made a selection for projectile particles based on the signals in the front ion chamber. Events to be accepted were required to have signals in the two halves of the chamber within 2 standard deviations of the peak of the projectile signals. In the worst case, that of a high-energy gold projectile, this corresponded to a value of $0.92 \mathrm{cu}$. In this case, the probability that a particle with $|\Delta Z|=1$ would pass the selection in both halves of the detector is 0.19 , while the probability of a particle with $|\Delta Z|=2$ passing is vanishingly small. For the best case, that of an iron projectile, the 2-standard-deviation requirement corresponds to 0.15 $\mathrm{cu}$, and essentially no particles passing the selection are of different charge than that of the projectile. In order for an event in which two or more nuclei entered together to pass, the particles would have to have a fortuitous combination of charges so as to mimic the signal of a single projectile particle. Examination of the data indicates that the probability of such events is negligible.

Three runs with high-energy lanthanum projectiles were an exception. During these runs, the protective cover on the thin window to the vacuum of the particle accelerator was inadvertently left in place. This cover was a piece of $1.905 \mathrm{~cm}$ plywood. The thickness and density of the cover was equivalent to $\approx 1.5 \mathrm{~g} / \mathrm{cm}^{2}$ of polyethylene, or 0.3 interaction length for lanthanum. The projectiles lost about $75 \mathrm{Mev} /$ nucleon to ionization energy losses while passing through the plywood. However, there was no indication in the appearance of the data or in the final results that, after the appropriate corrections, the plywood runs were unusual, and we have included the results from these runs in our analysis, because they were at a unique energy.

\section{Selection for noninteraction in the detector}

The Cherenkov detectors had thick enough radiators to cause $10 \%$ to $20 \%$ of the projectile nuclei and fragments to interact. In order to select those particles which did not interact in the detectors we required consistency between the ion counters before and after the Cherenkov detector. The signal in the ion counter just behind the target holder position (I-2) and the sum of the signals of the ion counters following the Cherenkov detector (I-3 and I-4) were required to be consistent within 2 standard deviations. This noninteraction selection excluded many of the events with $|\Delta Z|= \pm 1$ occurring in the detector, most of those with $\Delta Z=-2$, and essentially all with $|\Delta Z|>2$. 


\section{The targets}

The choice of target thickness was governed by a number of conflicting requirements. They should be thin to minimize energy loss and multiple interactions; but thick to maximize the efficiency of the limited running time available, to minimize the relative importance of corrections for overlap of the signals from the large projectile peak with the small peaks due to fragments with small $|\Delta Z|$, and to minimize the relative importance of corrections for particles interacting in the material of the detector. In general, targets used in this run were somewhat thicker than those used previously, e.g., about 0.32 mean interaction length for lanthanum on polyethylene as opposed to about 0.21 for xenon on polyethylene. This means that although the statistical uncertainties for each charge peak were decreased, and the importance of background corrections was reduced, the correction for multiple interactions in a thick target was more critical than in the analysis of the experiment of Ref. 13. We chose the thicknesses of the different targets so that in general the energy loss was $\approx 100 \mathrm{MeV} /$ nucleon, and thus was significantly less than the energy differences between different runs. This had the consequence that for comparable exposures fewer fragments were produced in the heavier targets. Table II lists the thicknesses of targets used for each projectile-energy-target combination.
We used carbon (C), polyethylene $\left(\mathrm{CH}_{2}\right)$, aluminum, $(\mathrm{Al})$, copper $(\mathrm{Cu})$, and, for the iron projectiles only, lead targets. The cross sections for the fragmentation of a projectile on targets of carbon $\left(\sigma_{\mathrm{C}}\right)$ and the average cross sections per nucleus of polyethylene $\left(\sigma_{\mathrm{CH}_{2}}\right)$ could be used to obtain the cross sections on a hydrogen target by subtraction

$$
\sigma_{\mathrm{H}}=0.5\left(3 \sigma_{\mathrm{CH}_{2}}-\sigma_{\mathrm{C}}\right) \text {. }
$$

The interactions with hydrogen are of astrophysical interest because hydrogen is the dominant component of the interstellar medium. From a nuclear-physics viewpoint they can be compared with fragmentation resulting from proton bombardment of heavy targets. Carbon also provided a sample of a light target nucleus. Aluminum is an important component of the material surrounding many particle detectors in space and balloon experiments, so a detailed knowledge of its cross section permits better correction for the interactions of cosmic rays in this material. It was also a sample of a mediummass nucleus. Copper was a sample of a moderately heavy nucleus. There was insufficient running time to permit the use of really heavy target nuclei, which require significantly longer exposure times.

TABLE II. Targets, energies, projectiles, and numbers of particles.

\begin{tabular}{|c|c|c|c|c|c|c|c|}
\hline $\begin{array}{c}\text { Energy at } \\
\text { top of target } \\
\text { (MeV/nucleon) }\end{array}$ & $\begin{array}{c}\text { Target } \\
\text { type }\end{array}$ & $\mathrm{cm}$ & $\begin{array}{r}\text { Target } \\
\left(\mathrm{g} / \mathrm{cm}^{2}\right) \\
\end{array}$ & $\begin{array}{l}\text { thickness } \\
\text { (interaction } \\
\text { length) }\end{array}$ & $\begin{array}{c}\text { Energy loss } \\
\text { of beam } \\
\text { (MeV/nucleon) }\end{array}$ & $\begin{array}{c}\text { Number of incident } \\
\text { beam particles }\end{array}$ & $\begin{array}{l}\text { Number of particles } \\
\text { breaking up and } \\
\text { not triggering (estimate) }\end{array}$ \\
\hline \multicolumn{8}{|c|}{ Gold beam } \\
\hline 618 & $\mathrm{CH}_{2}$ & 1.346 & 1.252 & 0.3031 & 118 & $225057 \pm 482$ & 17782 \\
\hline 723 & $\mathrm{CH}_{2}$ & 1.346 & 1.252 & 0.3031 & 110 & $191909 \pm 438$ & 9206 \\
\hline 821 & $\mathrm{CH}_{2}$ & 1.346 & 1.252 & 0.3031 & 106 & $170879 \pm 413$ & 5204 \\
\hline 968 & $\mathrm{CH}_{2}$ & 1.346 & 1.252 & 0.3031 & 101 & $161505 \pm 401$ & 4039 \\
\hline 618 & C & 0.826 & 1.520 & 0.2063 & 113 & $164515 \pm 416$ & 8702 \\
\hline 723 & $\mathrm{C}$ & 0.826 & 1.520 & 0.2063 & 105 & $178984 \pm 424$ & 5426 \\
\hline 821 & $\mathrm{C}$ & 0.826 & 1,520 & 0.2063 & 102 & $155523 \pm 394$ & 1726 \\
\hline 968 & $\mathrm{C}$ & 0.826 & 1.520 & 0.2063 & 97 & $176944 \pm 419$ & 1248 \\
\hline 618 & Al & 0.635 & 1.715 & 0.1227 & 113 & $274265 \pm 532$ & 11156 \\
\hline 723 & Al & 0.635 & 1.715 & 0.1227 & 106 & $272783 \pm 523$ & 5655 \\
\hline 821 & Al & 0.635 & 1.715 & 0.1227 & 103 & $254513 \pm 503$ & 3903 \\
\hline 968 & Al & 0.635 & 1.715 & 0.1227 & 97 & $275888 \pm 524$ & 4412 \\
\hline 618 & $\mathrm{Cu}$ & 0.216 & 1.929 & 0.0752 & 111 & $478844 \pm 704$ & 14126 \\
\hline 723 & $\mathrm{Cu}$ & 0.216 & 1.929 & 0.0752 & 104 & $443607 \pm 666$ & 2146 \\
\hline 821 & $\mathrm{Cu}$ & 0.216 & 1.929 & 0.0752 & 101 & $420128 \pm 646$ & 4879 \\
\hline 968 & $\mathrm{Cu}$ & 0.216 & 1.929 & 0.0752 & 97 & $351776 \pm 591$ & 3398 \\
\hline \multicolumn{8}{|c|}{ Holmium beam } \\
\hline 548 & $\mathrm{CH}_{2}$ & 1.549 & 1.441 & 0.3176 & 124 & $120888 \pm 348$ & 10764 \\
\hline 822 & $\mathrm{CH}_{2}$ & 1.549 & 1.441 & 0.3176 & 104 & $119759 \pm 345$ & 4610 \\
\hline 976 & $\mathrm{CH}_{2}$ & 1.346 & 1.252 & 0.2759 & 87 & $143464 \pm 378$ & 5673 \\
\hline 548 & $\mathrm{C}$ & 0.953 & 1.754 & 0.2198 & 119 & $167745 \pm 411$ & 17111 \\
\hline 822 & $\mathrm{C}$ & 0.953 & 1.754 & 0.2198 & 100 & $154947 \pm 393$ & 2635 \\
\hline 976 & $\mathrm{C}$ & 0.826 & 1.520 & 0.1905 & 83 & $135321 \pm 367$ & 4825 \\
\hline 548 & Al & 0.736 & 1.987 & 0.1321 & 119 & $180416 \pm 426$ & 19790 \\
\hline 822 & Al & 0.736 & 1.987 & 0.1321 & 101 & $244149 \pm 494$ & 4443 \\
\hline 822 & $\mathrm{Cu}$ & 0.254 & 2.268 & 0.0827 & 101 & $389816 \pm 624$ & 3896 \\
\hline
\end{tabular}


TABLE II. (Continued).

\begin{tabular}{|c|c|c|c|c|c|c|c|}
\hline \multicolumn{8}{|c|}{ Lanthanum beam } \\
\hline 543 & $\mathrm{CH}_{2}$ & 0.838 & 0.779 & 0.1567 & 66 & $171950 \pm 414$ & 7986 \\
\hline 675 & $\mathrm{CH}_{2}$ & 1.829 & 1.761 & 0.3548 & 118 & $133431 \pm 365$ & 10927 \\
\hline 828 & $\mathrm{CH}_{2}$ & 1.829 & 1.761 & 0.3548 & 110 & $139483 \pm 373$ & 9582 \\
\hline 956 & $\mathrm{CH}_{2}$ & 1.829 & 1.761 & 0.3548 & 105 & $116427 \pm 341$ & 7681 \\
\hline $1130^{\mathrm{a}}$ & $\mathrm{CH}_{2}$ & 1.829 & 1.761 & 0.3548 & 102 & $69282 \pm 263$ & 4554 \\
\hline 1214 & $\mathrm{CH}_{2}$ & 1.829 & 1.761 & 0.3548 & 100 & $99922 \pm 316$ & 6979 \\
\hline 543 & $\mathrm{C}$ & 0.508 & 0.935 & 0.1087 & 63 & $152187 \pm 390$ & 4901 \\
\hline 675 & $\mathrm{C}$ & 1.118 & 2.057 & 0.2391 & 109 & $114489 \pm 338$ & 5193 \\
\hline 828 & $\mathrm{C}$ & 1.118 & 2.057 & 0.2391 & 102 & $124939 \pm 353$ & 6065 \\
\hline 956 & $\mathrm{C}$ & 1.118 & 2.057 & 0.2391 & 97 & $114541 \pm 338$ & 5708 \\
\hline $1130^{\mathrm{a}}$ & $\mathrm{C}$ & 1.118 & 2.057 & 0.2391 & 95 & $98932 \pm 314$ & 5402 \\
\hline 1214 & $\mathrm{C}$ & 1.118 & 2.057 & 0.2391 & 93 & $135621 \pm 368$ & 7698 \\
\hline 543 & $\mathrm{Al}$ & 0.406 & 1.096 & 0.068 & 65 & $178184 \pm 422$ & 4674 \\
\hline 675 & $\mathrm{Al}$ & 0.889 & 2.400 & 0.1489 & 113 & $195250 \pm 441$ & 7981 \\
\hline 828 & Al & 0.889 & 2.400 & 0.1489 & 106 & $181761 \pm 426$ & 7838 \\
\hline 956 & Al & 0.889 & 2.400 & 0.1489 & 101 & $177779 \pm 421$ & 7507 \\
\hline $1130^{\mathrm{a}}$ & $\mathrm{Al}$ & 0.889 & 2.400 & 0.1489 & 98 & $132878 \pm 364$ & 5598 \\
\hline 1214 & $\mathrm{Al}$ & 0.889 & 2.400 & 0.1489 & 96 & $215963 \pm 464$ & 9226 \\
\hline 543 & $\mathrm{Cu}$ & 0.140 & 1.250 & 0.0428 & 65 & $139599 \pm 373$ & 2209 \\
\hline 675 & $\mathrm{Cu}$ & 0.305 & 2.724 & 0.0933 & 112 & $385038 \pm 620$ & 12097 \\
\hline 828 & $\mathrm{Cu}$ & 0.305 & 2.724 & 0.0933 & 105 & $354385 \pm 595$ & 9444 \\
\hline 951 & $\mathrm{Cu}$ & 0.305 & 2.724 & 0.0933 & 101 & $340518 \pm 583$ & 9823 \\
\hline 1214 & $\mathrm{Cu}$ & 0.305 & 2.724 & 0.0933 & 96 & $259493 \pm 509$ & 6521 \\
\hline \multicolumn{8}{|c|}{ Iron beam } \\
\hline 1593 & $\mathrm{C}$ & 1.944 & 3.577 & 0.2848 & 46 & $137752 \pm 371$ & 10007 \\
\hline 1593 & $\mathrm{Al}$ & 1.524 & 4.115 & 0.1806 & 48 & $194472 \pm 441$ & 11026 \\
\hline 1593 & $\mathrm{Cu}$ & 0.521 & 4.653 & 0.1163 & 48 & $333967 \pm 577$ & 12580 \\
\hline 1593 & $\mathrm{~Pb}$ & 0.655 & 7.450 & 0.0886 & 60 & $377967 \pm 614$ & 12853 \\
\hline
\end{tabular}

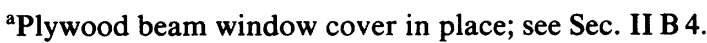

\section{DETERMINATION OF CROSS SECTIONS}

\section{A. Introduction}

The number of particles of each charge leaving the targets was determined from a combination of the signals from the Cherenkov and iron chambers; e.g., Fig. 2, see Sec III B. The data were reduced to histograms of the number of events per combined signal level, with these signal levels being nearly proportional to the square of the charge of the largest fragment; e.g., Fig. 3. These histograms have distinct peaks, nearly Gaussian in shape, corresponding to each charge from that of the projectile nuclei $Z_{\text {proj }}$ down to $|\Delta Z| \approx 0.5 Z_{\text {proj }}$. Clearly it is not difficult to resolve the individual fragments from data of this quality.

The signal level for any one event was formed from the combined signals of all the individual fragments of the projectile nucleus exiting the target and was proportional to the sum of the squares of the charges of the particles. Except in the case of the gold projectile, the largest fragment always had a much higher charge than any of the other fragments for $|\Delta Z| \leq 50 \%$ of $Z_{\text {prog. }}$. For still larger $|\Delta Z|$, the effect of the other lighter fragments became important enough to smear the resolution of individual charge peaks. For gold, which has a significant probabili-

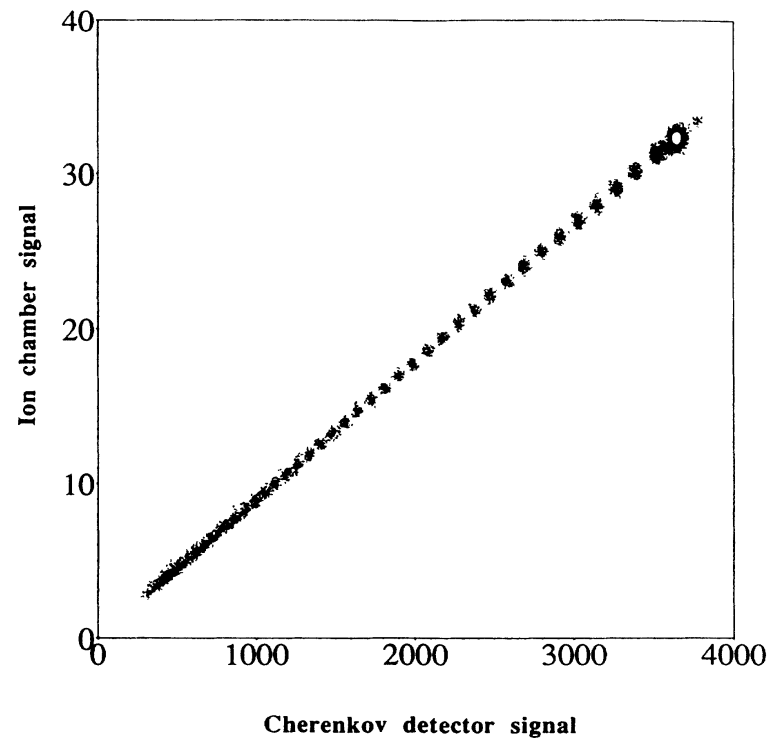

FIG. 2. Plot of the sum of ionization counter signals versus the sum of the Cherenkov detector signals for 1165 $\mathrm{MeV} /$ nucleon $\mathrm{La}$ on polyethylene. The total number of events shown is 22777 , while 47537 are excluded in the ellipse around the projectile peak. Each dot may represent more than one event. 


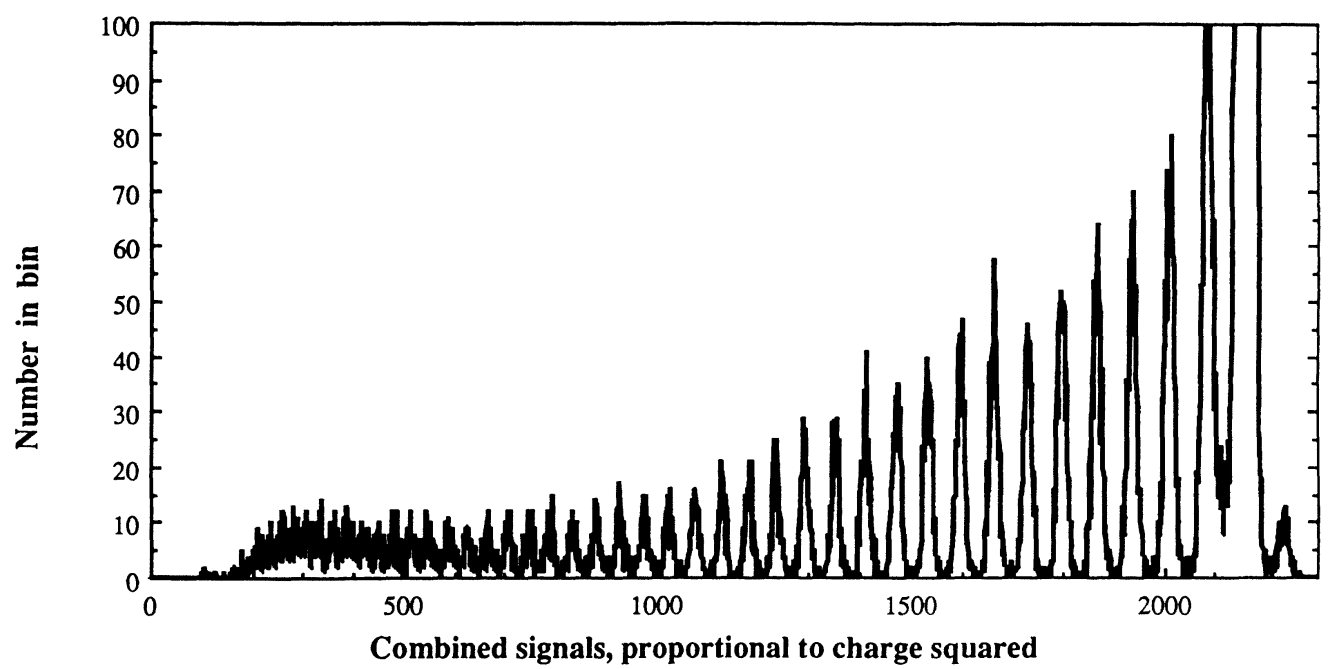

FIG. 3. Histogram of combined ion chamber and Cherenkov signals for $1165 \mathrm{MeV} /$ nucleon La on polyethylene from Fig. 2. Maximum peak height of 2260 .

ty for undergoing fission, ${ }^{19}$ the two fragments produced by events with symmetric fission had a combined signal at a nominal $\Delta Z \approx-23$, spreading up to slightly less negative values of $\Delta Z$ as the fission becomes more asymmetric, and spreading broadly down as the number of subsidiary fragments increases ("dirty" fission). The peaks due to individual fragments in this charge region, although still resolvable, consequently had an additional component of fission interactions which produced a continuous background. These background events were counted along with the events in the corresponding charge peaks, and are reflected in apparent excess cross sections when compared to the general trend of the cross sections with $\Delta Z$. The specific cross sections that can be derived for the fission process will be discussed in the

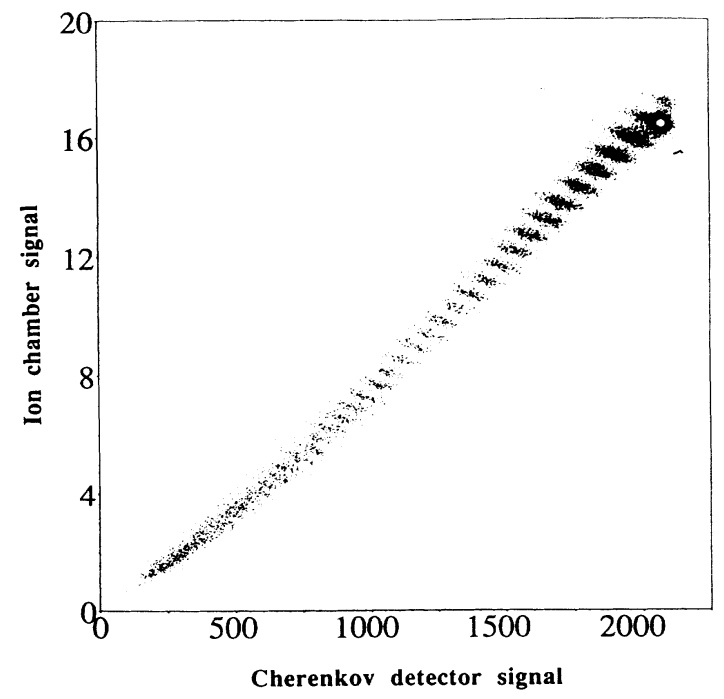

FIG. 4. The sum of ionization counter signals versus the sum of the Cherenkov detector signals for a $621 \mathrm{MeV} /$ nucleon $\mathrm{La}$ projectile on a polyethylene target. companion paper. ${ }^{1}$

The counts observed in the charge peak are for those events where the particles did not interact in the material of the detector. To find the numbers of particles of each charge produced in the target we corrected for those that did interact, by using the total charge-changing interaction cross sections from Eq. (1). We also corrected for the effects of secondary interactions where particles interacted more than once while passing through the target. This "thick-target correction" is discussed in Sec. III C 3. Cross sections for the production of nuclei of each $\Delta Z$ were determined by comparison of the corrected numbers of particles of each charge produced in the target to the number of entering projectile particles. This number was deduced for each run from the number of

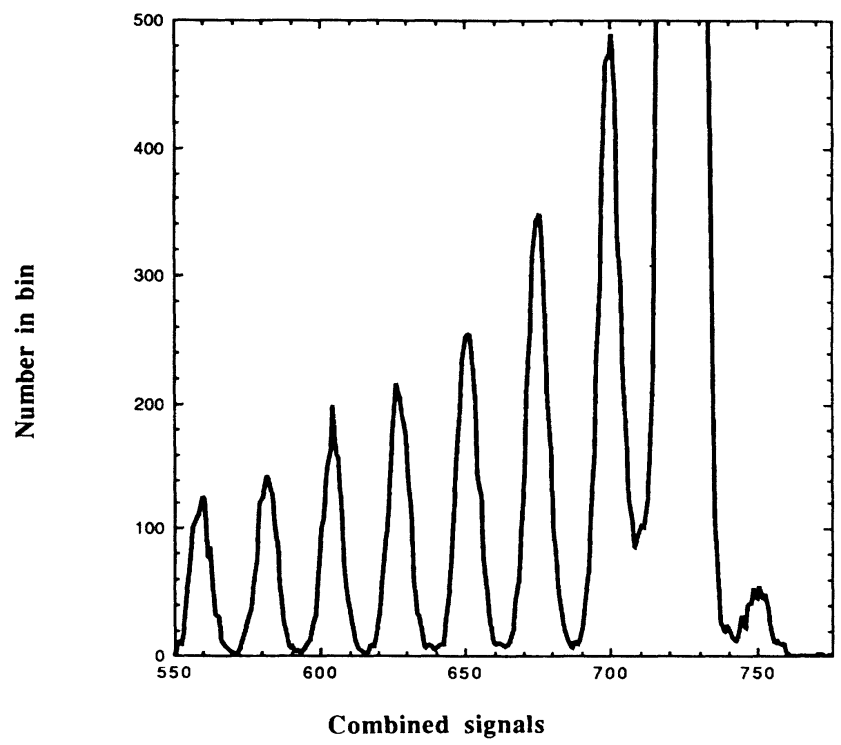

FIG. 5. Expanded histogram of combined signals from Fig. 4 , showing the peaks from $\Delta Z=+1$ to -6 . 
surviving particles and the total cross sections for charge-changing interactions in the detector and in the target, as described in Secs. II B 1 and III C 2.

\section{B. Signals}

For each run we constructed a charge histogram from a combination of the Cherenkov and ion chamber signals in the instrument. To combine the individual ion chamber and Cherenkov counter signals into single signals, we used weighting factors which gave the best ratio of signal to dispersion for the total signal.

There were considerable variation in dispersion, as measured in cu, between the ion counter and Cherenkov signals from projectile to projectile and energy to energy. The Cherenkov signals were greatly reduced at lower energies, with larger relative photon statistical fluctuations, while the ion chamber signals were somewhat increased. At the higher energies, neither type of detector was very sensitive to energy and thus, at these energies the best charge resolution is obtained by combining the signals weighted according to the standard deviation for each. At lower energies, the best charge resolution is obtained by combining the signals with weighting found from the energy response of the two types of detectors. In practice, the weighting was found from the scatter plots of ion counter signals versus Cherenkov signals, e.g., Fig. 4, by determining the slopes of the major axes of the roughly elliptical distribution observed on these plots for each charge. The resolution obtained, Fig. 5, is not significantly improved by slight variations of the weighting from those found by eye and straight edge, so a simple visual fit was used to determine the slopes of the axes. When there was no target only a few particles interacted in the small amount of material between I-1 and I-2, hence, the slopes of the ellipse axes in the target-out runs are not well defined. Instead, the weighting for target-in runs was used for the corresponding target-out runs.

TABLE III. Corrections for $\mathrm{La}$ on $\mathrm{CH}_{2}$ at $1163 \mathrm{MeV} /$ nucleon.

\begin{tabular}{|c|c|c|c|c|c|c|c|}
\hline$\Delta Z$ & $\begin{array}{l}\text { Number in } \\
\text { Number } \\
\text { counted }\end{array}$ & $\begin{array}{l}\text { beam }=99 \\
\text { Target-out } \\
\text { correction }\end{array}$ & $\begin{array}{l}2 \pm 316 \\
\text { Number } \\
\text { in peak }\end{array}$ & $\begin{array}{l}\text { Number at } \\
\text { top of detector }\end{array}$ & $\begin{array}{l}\text { Total cross section } \\
\text { First estimate of } \\
\text { cross section }(\mathrm{mb})\end{array}$ & $\begin{array}{l}=1682.6 \mathrm{mb} \\
\text { Thick-target } \\
\text { correction }(\mathrm{mb})\end{array}$ & $\begin{array}{c}\text { Cross section } \\
(\mathrm{mb})\end{array}$ \\
\hline 1 & & & $238.8 \pm 16.4$ & $308.2 \pm 18.4$ & 16.3 & $3.6 \pm 0.7$ & $19.9 \pm 1.4$ \\
\hline 0 & & & $52871.3 \pm 229.5$ & $68176.0 \pm 260.7$ & & & \\
\hline-1 & & & $2443.2 \pm 52.7$ & $3147.3 \pm 59.0$ & 166.8 & $36.2 \pm 2.4$ & $203.0 \pm 4.5$ \\
\hline-2 & & & $1579.8 \pm 40.2$ & $2024.9 \pm 45.4$ & 107.3 & $18.3 \pm 1.8$ & $125.6 \pm 3.3$ \\
\hline-3 & 1333.0 & 12.1 & $1320.9 \pm 36.6$ & $1691.4 \pm 41.4$ & 89.6 & $13.3 \pm 1.6$ & $103.0 \pm 3.0$ \\
\hline-4 & 1148.6 & 9.2 & $1139.3 \pm 34.0$ & $1451.4 \pm 38.3$ & 76.9 & $9.5 \pm 1.4$ & $86.5 \pm 2.7$ \\
\hline-5 & 1120.0 & 7.5 & $1112.5 \pm 33.6$ & $1414.3 \pm 37.8$ & 75.0 & $8.6 \pm 1.4$ & $83.6 \pm 2.6$ \\
\hline-6 & 909.3 & 6.4 & $902.9 \pm 30.2$ & $1143.1 \pm 34.0$ & 60.6 & $4.9 \pm 1.2$ & $65.4 \pm 2.3$ \\
\hline-7 & 940.7 & 5.5 & $935.2 \pm 30.7$ & $1180.3 \pm 34.5$ & 62.6 & $5.2 \pm 1.2$ & $67.7 \pm 2.3$ \\
\hline-8 & 812.0 & 4.9 & $807.1 \pm 28.6$ & $1015.5 \pm 32.0$ & 53.8 & $3.0 \pm 1.1$ & $56.8 \pm 2.1$ \\
\hline-9 & 729.0 & 4.4 & $724.6 \pm 27.1$ & $908.7 \pm 30.3$ & 48.2 & $1.8 \pm 1.0$ & $50.0 \pm 1.9$ \\
\hline-10 & 649.0 & 4.0 & $645.0 \pm 25.5$ & $805.4 \pm 28.5$ & 42.7 & $0.8 \pm 0.9$ & $43.4 \pm 1.8$ \\
\hline-11 & 588.0 & 3.6 & $584.4 \pm 24.3$ & $728.1 \pm 27.1$ & 38.6 & $0.2 \pm 0.8$ & $38.7 \pm 1.7$ \\
\hline-12 & 512.0 & 3.3 & $508.7 \pm 22.7$ & $631.6 \pm 25.2$ & 33.5 & $-0.6 \pm 0.8$ & $32.9 \pm 1.5$ \\
\hline-13 & 471.2 & 3.1 & $468.1 \pm 21.8$ & $579.3 \pm 24.2$ & 30.7 & $-0.8 \pm 0.7$ & $29.9 \pm 1.4$ \\
\hline-14 & 380.2 & 2.9 & $377.3 \pm 19.6$ & $465.9 \pm 21.7$ & 24.7 & $-1.7 \pm 0.6$ & $23.0 \pm 1.2$ \\
\hline-15 & 328.6 & 2.7 & $325.9 \pm 18.2$ & $401.3 \pm 20.2$ & 21.3 & $-1.8 \pm 0.6$ & $19.4 \pm 1.1$ \\
\hline-16 & 286.9 & 2.6 & $284.3 \pm 17.0$ & $348.1 \pm 18.8$ & 18.5 & $-2.0 \pm 0.5$ & $16.5 \pm 1.0$ \\
\hline-17 & 277.1 & 2.4 & $274.7 \pm 16.7$ & $334.6 \pm 18.4$ & 17.7 & $-1.7 \pm 0.5$ & $16.1 \pm 1.0$ \\
\hline-18 & 251.0 & 2.3 & $248.7 \pm 15.9$ & $302.1 \pm 17.5$ & 16.0 & $-1.7 \pm 0.5$ & $14.3 \pm 1.0$ \\
\hline-19 & 246.2 & 2.2 & $244.0 \pm 15.7$ & $295.6 \pm 17.3$ & 15.7 & $-1.4 \pm 0.5$ & $14.3 \pm 1.0$ \\
\hline-20 & 234.3 & 2.1 & $232.2 \pm 15.4$ & $279.9 \pm 16.8$ & 14.8 & $-1.3 \pm 0.4$ & $13.5 \pm 0.9$ \\
\hline-21 & 213.5 & 2.0 & $211.5 \pm 14.7$ & $254.3 \pm 16.1$ & 13.5 & $-1.3 \pm 0.4$ & $12.1 \pm 0.9$ \\
\hline-22 & 197.8 & 1.9 & $195.9 \pm 14.1$ & $234.2 \pm 15.4$ & 12.4 & $-1.3 \pm 0.4$ & $11.1 \pm 0.8$ \\
\hline-23 & 200.3 & 1.8 & $198.5 \pm 14.2$ & $236.4 \pm 15.5$ & 12.5 & $-1.1 \pm 0.4$ & $11.4 \pm 0.9$ \\
\hline-24 & 177.8 & 1.8 & $176.0 \pm 13.4$ & $208.7 \pm 14.6$ & 11.1 & $-1.2 \pm 0.4$ & $9.8 \pm 0.8$ \\
\hline-25 & 196.3 & 1.7 & $194.6 \pm 14.1$ & $230.3 \pm 15.3$ & 12.2 & $-0.9 \pm 0.4$ & $11.3 \pm 0.9$ \\
\hline-26 & 171.8 & 1.6 & $170.2 \pm 13.2$ & $199.9 \pm 14.2$ & 10.6 & $-1.1 \pm 0.4$ & $9.5 \pm 0.8$ \\
\hline-27 & 170.5 & 1.6 & $168.9 \pm 13.1$ & $197.5 \pm 14.2$ & 10.5 & $-1.0 \pm 0.3$ & $9.5 \pm 0.8$ \\
\hline-28 & 184.6 & 1.5 & $183.1 \pm 13.6$ & $213.4 \pm 14.7$ & 11.3 & $-0.8 \pm 0.4$ & $10.5 \pm 0.8$ \\
\hline-29 & 177.1 & 1.5 & $175.6 \pm 13.3$ & $203.5 \pm 14.4$ & 10.8 & $-0.9 \pm 0.4$ & $9.9 \pm 0.8$ \\
\hline-30 & 189.6 & 1.4 & $188.2 \pm 13.8$ & $217.6 \pm 14.8$ & 11.5 & $-0.7 \pm 0.4$ & $10.8 \pm 0.8$ \\
\hline-31 & 163.7 & 1.4 & $162.3 \pm 12.8$ & $186.8 \pm 13.8$ & 9.9 & $-1.0 \pm 0.3$ & $9.0 \pm 0.7$ \\
\hline-32 & 167.3 & 1.4 & $165.9 \pm 13.0$ & $189.4 \pm 13.8$ & 10.0 & $-0.9 \pm 0.3$ & $9.2 \pm 0.8$ \\
\hline
\end{tabular}


TABLE IV. Corrections for $\mathrm{Au}$ on $\mathrm{Cu}$ at $559 \mathrm{MeV} /$ nucleon.

\begin{tabular}{|c|c|c|c|c|c|c|c|}
\hline$\Delta \boldsymbol{Z}$ & $\begin{array}{l}\text { Number in } \\
\text { Number } \\
\text { counted }\end{array}$ & $\begin{array}{c}\text { beam }=4788 \\
\text { Target-out } \\
\text { correction }\end{array}$ & $\begin{array}{r}4.4 \pm 704.2 \\
\text { Number } \\
\text { in peak }\end{array}$ & $\begin{array}{c}\text { Number at } \\
\text { top of detector }\end{array}$ & $\begin{array}{l}\text { Total cross section } \\
\text { First estimate of } \\
\text { cross section }(\mathrm{mb})\end{array}$ & $\begin{array}{c}=4116.9 \mathrm{mb} \\
\text { Thick-target } \\
\text { correction (mb) }\end{array}$ & $\begin{array}{c}\text { Cross section } \\
(\mathrm{mb})\end{array}$ \\
\hline 1 & & & $1892.5 \pm 93.5$ & $2709.3 \pm 97.7$ & 321.2 & $12.5 \pm 1.8$ & $333.7 \pm 12.2$ \\
\hline 0 & & & $311254.7 \pm 573.0$ & $444119.0 \pm 679.1$ & & & \\
\hline-1 & & & $4875.3 \pm 208.7$ & $6944.8 \pm 213.6$ & 823.3 & $31.7 \pm 2.8$ & $855.0 \pm 26.4$ \\
\hline-2 & & & $1256.7 \pm 52.5$ & $1785.6 \pm 57.3$ & 211.7 & $1.6 \pm 1.4$ & $213.3 \pm 7.0$ \\
\hline-3 & 996.9 & 115.6 & $881.3 \pm 36.0$ & $1250.1 \pm 40.8$ & 148.2 & $2.3 \pm 1.2$ & $150.5 \pm 5.1$ \\
\hline-4 & 800.5 & 83.0 & $717.6 \pm 31.9$ & $1014.4 \pm 36.2$ & 120.3 & $1.8 \pm 1.0$ & $122.1 \pm 4.5$ \\
\hline-5 & 616.7 & 64.1 & $552.5 \pm 28.0$ & $779.8 \pm 31.8$ & 92.4 & $1.0 \pm 0.9$ & $93.5 \pm 3.9$ \\
\hline-6 & 604.5 & 52.0 & $552.6 \pm 27.2$ & $777.8 \pm 31.1$ & 92.2 & $1.3 \pm 0.9$ & $93.5 \pm 3.8$ \\
\hline-7 & 491.5 & 43.5 & $447.9 \pm 24.6$ & $628.9 \pm 28.0$ & 74.6 & $0.7 \pm 0.8$ & $75.2 \pm 3.5$ \\
\hline-8 & 495.8 & 37.3 & $458.5 \pm 24.3$ & $642.0 \pm 27.9$ & 76.1 & $0.9 \pm 0.8$ & $77.0 \pm 3.4$ \\
\hline-9 & 423.8 & 32.6 & $391.2 \pm 22.5$ & $546.8 \pm 25.8$ & 64.8 & $0.5 \pm 0.7$ & $65.3 \pm 3.2$ \\
\hline-10 & 400.5 & 28.8 & $371.7 \pm 21.8$ & $517.7 \pm 24.9$ & 61.4 & $0.5 \pm 0.7$ & $61.9 \pm 3.1$ \\
\hline-11 & 341.9 & 25.8 & $316.0 \pm 20.2$ & $439.4 \pm 23.1$ & 52.1 & $0.2 \pm 0.7$ & $52.3 \pm 2.8$ \\
\hline-12 & 367.8 & 23.4 & $344.4 \pm 20.7$ & $478.0 \pm 23.7$ & 56.7 & $0.5 \pm 0.7$ & $57.2 \pm 2.9$ \\
\hline-13 & 329.5 & 21.3 & $308.2 \pm 19.6$ & $426.6 \pm 22.4$ & 50.6 & $0.2 \pm 0.7$ & $50.8 \pm 2.8$ \\
\hline-14 & 275.4 & 19.6 & $255.8 \pm 18.1$ & $353.1 \pm 20.6$ & 41.9 & $0.0 \pm 0.6$ & $41.9 \pm 2.5$ \\
\hline-15 & 262.8 & 18.1 & $244.7 \pm 17.6$ & $336.9 \pm 20.0$ & 39.9 & $0.0 \pm 0.6$ & $39.9 \pm 2.4$ \\
\hline-16 & 257.4 & 16.8 & $240.6 \pm 17.3$ & $330.3 \pm 19.8$ & 39.2 & $0.1 \pm 0.6$ & $39.2 \pm 2.4$ \\
\hline-17 & 245.3 & 15.6 & $229.7 \pm 16.9$ & $314.2 \pm 19.2$ & 37.2 & $0.0 \pm 0.5$ & $37.3 \pm 2.4$ \\
\hline-18 & 272.7 & 14.6 & $258.1 \pm 17.6$ & $352.4 \pm 20.1$ & 41.8 & $0.2 \pm 0.6$ & $42.0 \pm 2.5$ \\
\hline-19 & 249.0 & 13.8 & $235.2 \pm 16.9$ & $320.2 \pm 19.2$ & 38.0 & $0.0 \pm 0.6$ & $38.0 \pm 2.3$ \\
\hline-20 & 238.9 & 13.0 & $225.9 \pm 16.5$ & $306.7 \pm 18.8$ & 36.4 & $0.0 \pm 0.5$ & $36.4 \pm 2.3$ \\
\hline-21 & 251.7 & 12.3 & $239.4 \pm 16.8$ & $323.7 \pm 19.2$ & 38.4 & $0.1 \pm 0.6$ & $38.5 \pm 2.3$ \\
\hline-22 & 242.7 & 11.6 & $231.1 \pm 16.5$ & $310.4 \pm 18.8$ & 36.8 & $0.0 \pm 0.5$ & $36.8 \pm 2.3$ \\
\hline-23 & 264.1 & 11.0 & $253.1 \pm 17.1$ & $338.0 \pm 19.4$ & 40.1 & $0.1 \pm 0.6$ & $40.2 \pm 2.4$ \\
\hline-24 & 269.1 & 10.5 & $258.6 \pm 17.2$ & $342.0 \pm 19.5$ & 40.5 & $0.1 \pm 0.6$ & $40.6 \pm 2.4$ \\
\hline-25 & 270.9 & 10.0 & $260.8 \pm 17.2$ & $343.2 \pm 19.5$ & 40.7 & $0.1 \pm 0.6$ & $40.8 \pm 2.4$ \\
\hline-26 & 248.1 & 9.6 & $238.5 \pm 16.5$ & $311.0 \pm 18.6$ & 36.9 & $-0.1 \pm 0.5$ & $36.8 \pm 2.3$ \\
\hline-27 & 265.9 & 9.2 & $256.7 \pm 17.0$ & $332.8 \pm 19.1$ & 39.5 & $0.0 \pm 0.5$ & $39.5 \pm 2.3$ \\
\hline-28 & 271.4 & 8.8 & $262.6 \pm 17.2$ & $338.0 \pm 19.2$ & 40.1 & $0.0 \pm 0.5$ & $40.1 \pm 2.3$ \\
\hline-29 & 243.0 & 8.4 & $234.6 \pm 16.3$ & $300.0 \pm 18.2$ & 35.6 & $-0.2 \pm 0.5$ & $35.4 \pm 2.2$ \\
\hline-30 & 235.5 & 8.1 & $227.4 \pm 16.0$ & $289.0 \pm 17.8$ & 34.3 & $-0.2 \pm 0.5$ & $34.1 \pm 2.2$ \\
\hline-31 & 287.3 & 7.8 & $279.5 \pm 17.5$ & $353.2 \pm 19.5$ & 41.9 & $0.1 \pm 0.6$ & $42.0 \pm 2.4$ \\
\hline-32 & 241.0 & 7.5 & $233.4 \pm 16.1$ & $293.0 \pm 17.9$ & 34.7 & $-0.3 \pm 0.5$ & $34.5 \pm 2.2$ \\
\hline-33 & 250.7 & 7.3 & $243.4 \pm 16.4$ & $304.1 \pm 18.2$ & 36.1 & $-0.2 \pm 0.5$ & $35.9 \pm 2.2$ \\
\hline-34 & 211.2 & 7.0 & $204.2 \pm 15.1$ & $253.8 \pm 16.7$ & 30.1 & $-0.3 \pm 0.5$ & $29.7 \pm 2.0$ \\
\hline-35 & 237.7 & 6.8 & $230.9 \pm 16.0$ & $285.4 \pm 17.6$ & 33.8 & $-0.2 \pm 0.5$ & $33.7 \pm 2.1$ \\
\hline-36 & 229.6 & 6.6 & $223.0 \pm 15.7$ & $274.5 \pm 17.3$ & 32.5 & $-0.2 \pm 0.5$ & $32.3 \pm 2.1$ \\
\hline
\end{tabular}

\section{Corrections}

Three main corrections have to be made to the raw data in order to find the true cross sections. Each of these corrections is discussed in the following sections. As examples of the magnitudes of these corrections Tables III and IV show those for lanthanum on a polyethylene target with an energy of $1163 \mathrm{MeV} /$ nucleon in the center of the target, and gold on a copper target with an energy of $559 \mathrm{MeV} /$ nucleon in the center of the target, respectively. We can see that the relative importance of the various corrections varies with projectile and $\Delta Z$. These are examples of some of the smallest and largest total uncertainties for heavy projectiles. Apart from the corrections to the top of the detector, which makes quite large changes in the individual numbers, but very small changes in the relative numbers, the corrections are smaller than the statistical uncertainties for most values of $\Delta Z$.

\section{Target-out correction}

By applying the same selections and corrections to the target-out runs as the target-in runs, we can correct for particles interacting in the material of the detector, nonprimary projectile particles, and other background particles which pass the selection criteria. The energies through most of the detector of the particles in these target-out runs were somewhat higher than those in the target-in runs at the same projectile energy, because of the ionization energy losses in the targets. Thus the relative sizes of the signals in the Cherenkov and ion counters are different between the two runs. We, therefore, have a choice as to which target-out run to use with a given group of target-in runs. Either we can use the target-out run taken at the same projectile setting and close in time, which presumably had similar contamination and energy distribution within the run, or a targetout run which more closely matched the energy of the 
target-in run after passing through the target space (entering ion counter I-2), and so had similar signals and dispersion in the various detectors. Because in every case the contamination should be almost completely eliminated by the front ion counter selection (Sec. II B 4) and the energy spread in the projectiles was small, we used the second choice whenever possible. A typical target-out run is shown in Fig. 6 and can be compared with Fig. 3. Details of the application of these corrections are given elsewhere. $^{24}$ In most cases, apart for small $|\Delta Z|$, the target-out correction was small compared to statistical fluctuations.

\section{Top of detector correction}

The corrections described above, when applied to the numbers of selected events, given the numbers of particles of each charge which were produced in the target and did not interact in the material of the detector. However, the proportion of particles interacting in the detector is dependent on the type of particle, and so was different for each $Z$. In order to obtain the true number of particles which interacted in the target, we had to correct for those interacting in the detector. In addition, although we had the number of particles in the projectile charge peak which did not change charge in the target nor in the material of the detector, we did not have the number of projectile particles entering the top of the tar- get. The entering number had to be determined from the surviving number. Therefore we needed the total charge-changing interaction probability due to the material in the detector for each charge peak, and also the total charge-changing interaction probability of the projectile in the target. The probability for interaction can be determined from the cross section $(\Sigma$ in $\mathrm{mb})$ and the thickness of material ( $x$ in $\mathrm{g} / \mathrm{cm}^{2}$ ). If $N_{0}$ is the true number of incident particles, determined from the number surviving, and $A_{\text {avg }}$ is the average mass number of the target,

$$
N_{\text {interact }}=N_{0}\left[1-\exp \left[-\frac{6.02 \times 10^{-4} x \Sigma}{A_{\text {avg }}}\right]\right] \text {. }
$$

For $\Sigma$ we used the total charge-changing cross sections as determined from Eq. (1), which is valid for all nuclei with $Z>26$, or Eq. (2) for those nuclei with $Z \leq 26$. These expressions have no energy dependence but it is not expected that the total cross sections should have any large energy dependence at these energies (see Sec. III E). Table II gives, for each projectile and energy, the numbers of particles that failed to trigger the system after interacting. It can be seen that these numbers are generally of the order of $5 \%$ of the numbers of incident particles. The statistical fluctuation in the number of particles interacting in the detector was added quadratically to the overall uncertainty.

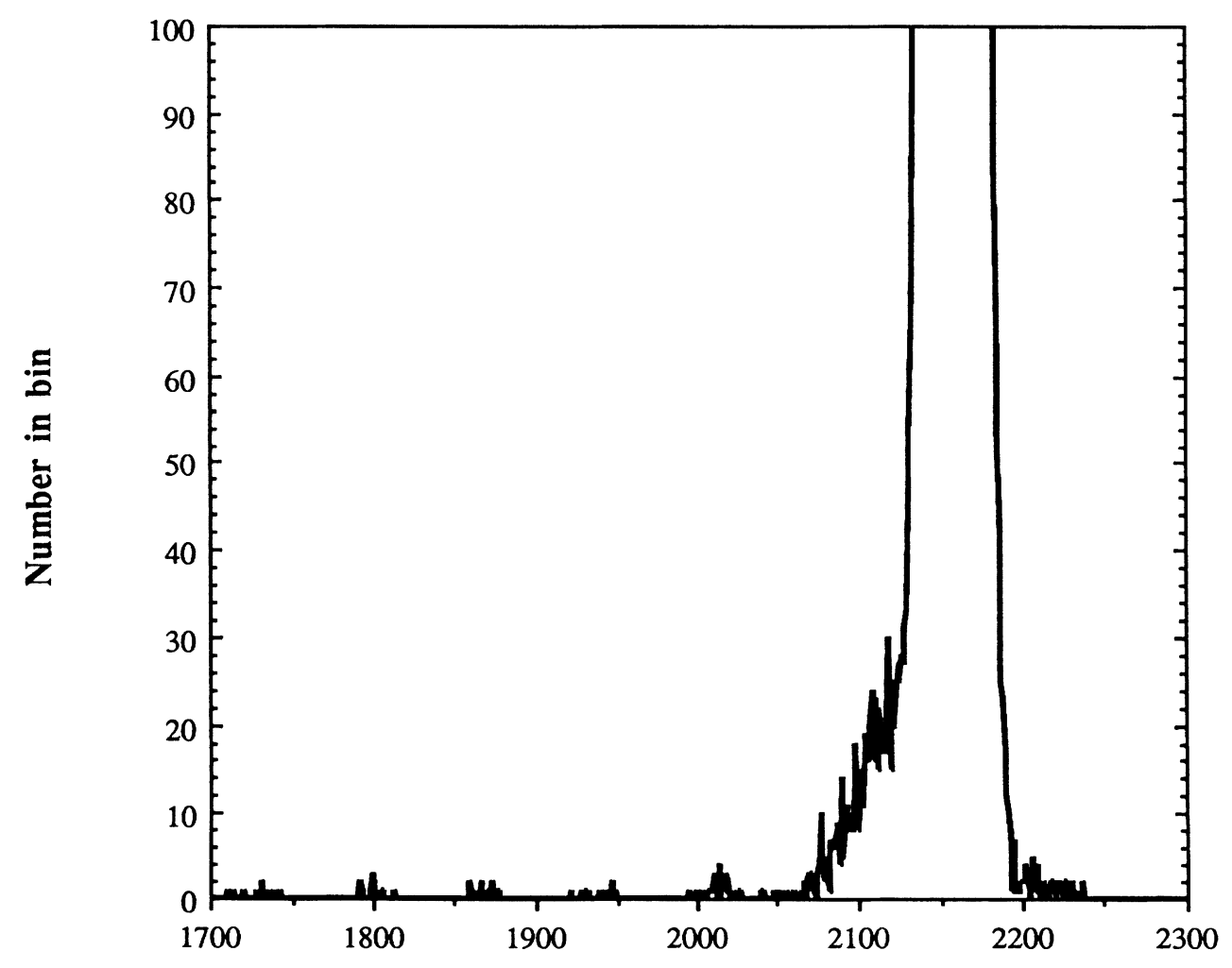

Combined signals

FIG. 6. Histograms for a target-out run of $1214 \mathrm{MeV} /$ nucleon La, showing the region corresponding to $\Delta Z=+2$ to -5 . Maximum peak height of 3152 . This figure may be compared with Fig. 3. 
In this analysis, it was necessary to assume that a given element has the standard distribution of stable isotopes, since both expressions for cross sections are dependent on the mass of the projectile. This assumption is not quite correct for most of the charge-changed fragments and for a number of those projectile charge particles that interact without changing charge, since, as we will show in Sec. IV, there appear to be considerably more neutrons than protons lost in these interactions. Moreover, the cross sections for particles which have just been produced in a peripheral nuclear interaction have been the subject of some controversy and could be appreciably different from those for normal, stable, nuclei. The "anomalon" phenomena, ${ }^{20-22}$ which suggested that some fragments and larger than normal cross sections shortly after production, has not been confirmed, at least in so far as it applies to heavy fragments. Hence we have assumed that these cross sections are not significantly different from those of normal nuclei. ${ }^{23}$

Calculating the difference in cross section for a nucleus of normal and stable mass and for a nucleus with a mass reduced by an amount consistent with the estimates of neutron loss in Sec. IV, gives us an estimate of the magnitude of the systematic uncertainty introduced by this assumption of stable fragments. For a $\Delta Z=-1$ fragment of ${ }_{57}^{137} \mathrm{La}$, such as ${ }_{56}^{137} \mathrm{Ba}$, the cross section in Lucite (Cherenkov radiator material) is $1720 \mathrm{mb}$ according to Eq. (1), while for ${ }_{56}^{130} \mathrm{Ba}$, which has lost seven neutrons, the cross section in Lucite is reduced by only $45 \mathrm{mb}$. So the probability for a charge-changing interaction in 1.6 $\mathrm{g} / \mathrm{cm}^{2}$ of Lucite varies from 0.220 to 0.215 between these two nuclei. The uncertainty introduced by our assumption is thus of the order $0.5 \%$. This was not included in the quoted uncertainties, but is small compared to the other uncertainties.

\section{Thick-target correction}

The targets we used in this experiment were not thin (see discussion in Sec. II C), in the sense that particles which interacted early in their passage through the target had an appreciable chance of suffering a second interaction. Thus the numbers in each charge peak reflect a smaller population of small charge changes and a larger population of large charge changes than would occur in a thin target.

To correct for this effect, the thickness of the target was considered in thin slabs of no more than 0.01 of the interaction length for the projectile nuclei. For each slab a computer program performed an iteration wherein a first guess for the partial cross section for the particular charge change gave the production rate of the given element, and the total cross section for charge change of that element from Eq. (1) or (2) (assuming normal, stable isotopes) gave the destruction rate of the existing numbers of that element. The deduced number was compared to the measured number of that element, and the initial guess was adjusted until these numbers differed by less than $0.1 \%$. This calculation assumed that the partial cross sections for production of a certain charge change are a constant fraction of the total charge-changing cross section, an approximately valid assumption. The uncertainties introduced by this correction were combined quadratically with the other uncertainties.

\section{Cross sections}

\section{Cross sections for negative charge change}

Here we show plots of cross section values $\sigma$ as a function of $\Delta Z$ for all negative values of $\Delta Z$, Figs. 7-13. For the pure targets the general power relationship between $\sigma$ and $\Delta Z$ is illustrated by using $\log -\log$ scales on these plots, apart for the case of the $\mathrm{Fe}$ projectile. For the measured $\mathrm{CH}_{2}$, and deduced hydrogen targets, the general exponential nature of the relationship is illustrated by using log-linear scales. Apart from these overall systematics, which will be discussed in more detail in our accompanying paper, there are certain specific features apparent to a greater or lesser degree. These include a noticeable odd-even effect for the iron cross sections, with a particularly strong peak at silicon, $\Delta Z=-12$, Fig. 7 . On the other hand, none of the heavier projectiles show any significant evidence for such an odd-even effect. The $\mathrm{La}$ plots appears to show, at least at low energies, a significantly enhanced cross section for $\mathrm{Sn}, \Delta Z=-7$, Fig. 8. Finally, and most well defined, is the fission peak for $\mathrm{Au}$, in the region of $22 \leq|\Delta Z| \leq 28$, Figs. 10 and 13 .

The complete set of cross sections found in this work and also the slightly modified cross sections found in the 1984 experiment $^{12}$ are listed in the thesis by Cummings ${ }^{24}$

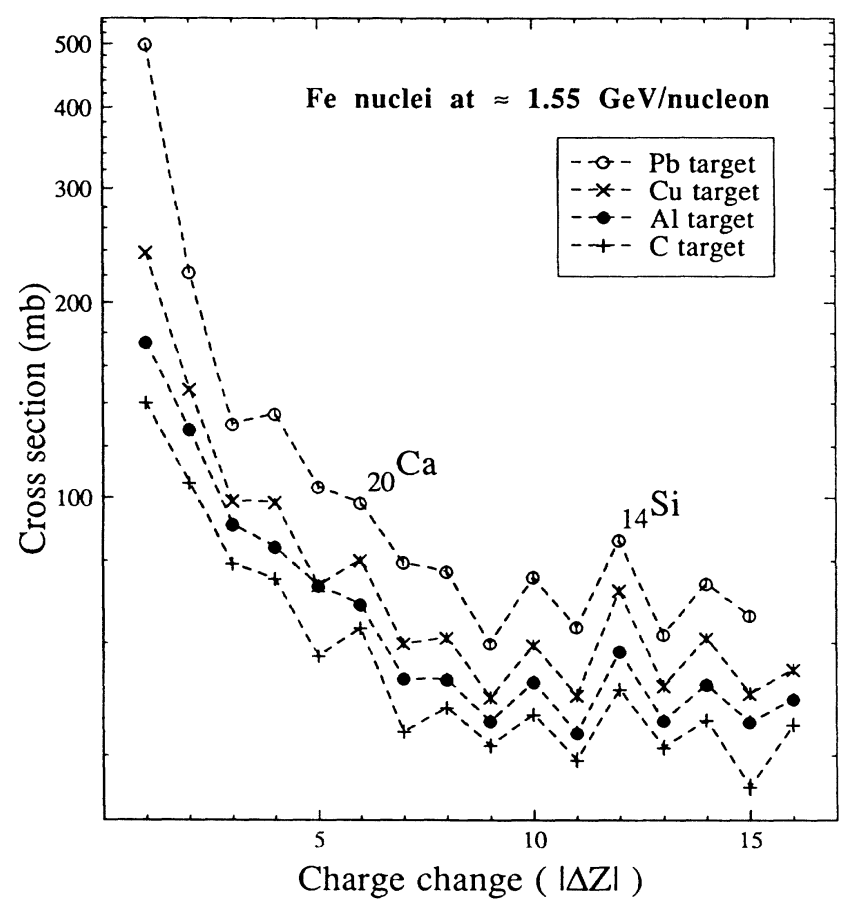

FIG. 7. Partial cross sections, in $\mathrm{mb}$, for $\mathrm{Fe}$ at 1569 $\mathrm{MeV} /$ nucleon on various targets as a function of $\Delta Z$. 


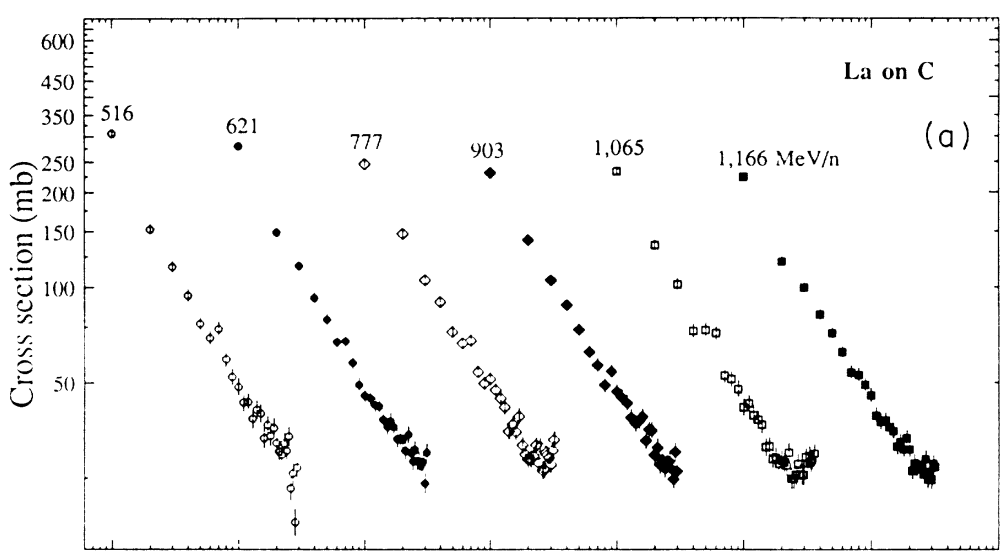

Charge change $(\Delta \mathrm{Z})$

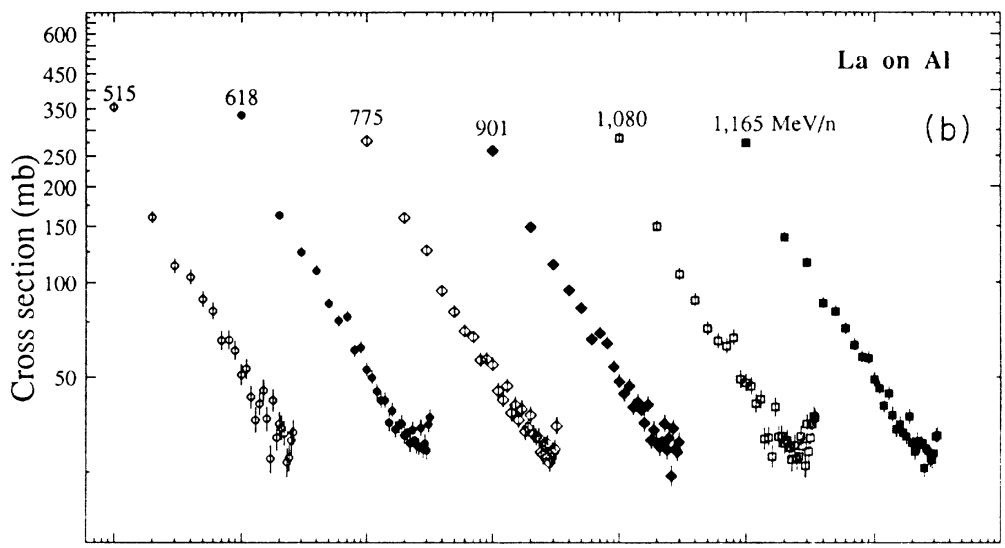

Charge change ( $\Delta \mathrm{Z})$

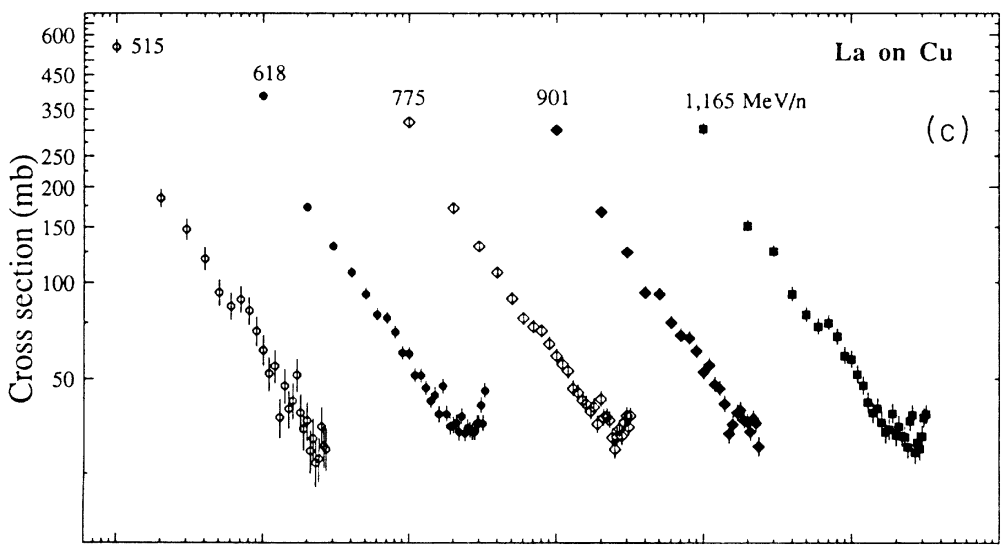

Charge change $(\Delta \mathrm{Z})$

FIG. 8. (a)-(c) Partial cross sections, in $\mathrm{mb}$, for $\mathrm{La}$ at six energies on $\mathrm{C}, \mathrm{Al}$, and $\mathrm{Cu}$ as a function of $\Delta Z$. Plotted on log-log scales, each energy run is displaced by a multiplicative factor from the preceding run for clarity, with the highest-energy run furthest displaced to the right. The individual energies are listed in Table II. 


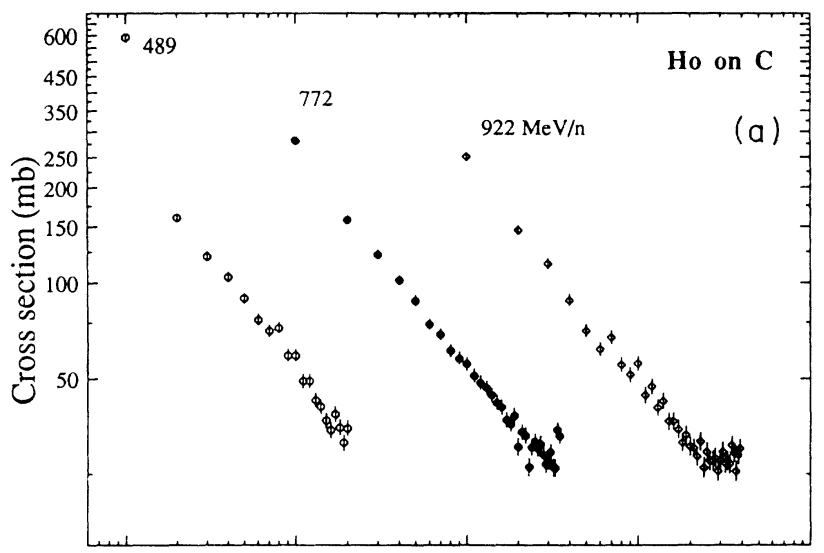

Charge change $(\Delta \mathrm{Z})$

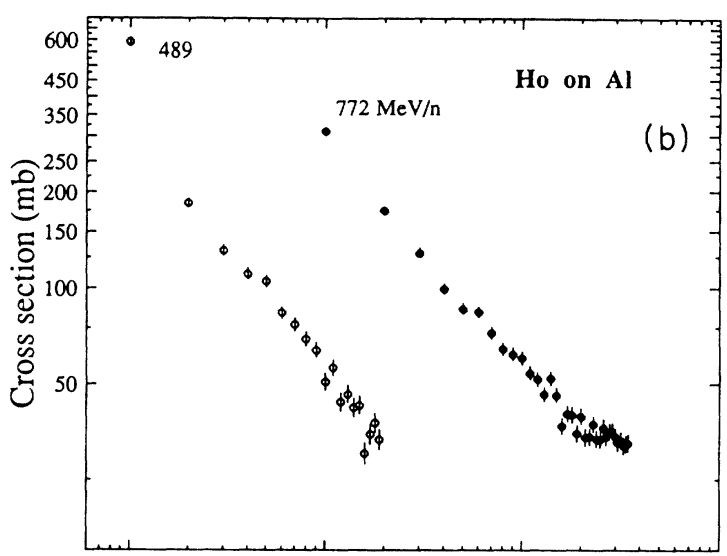

Charge change $(\Delta Z)$

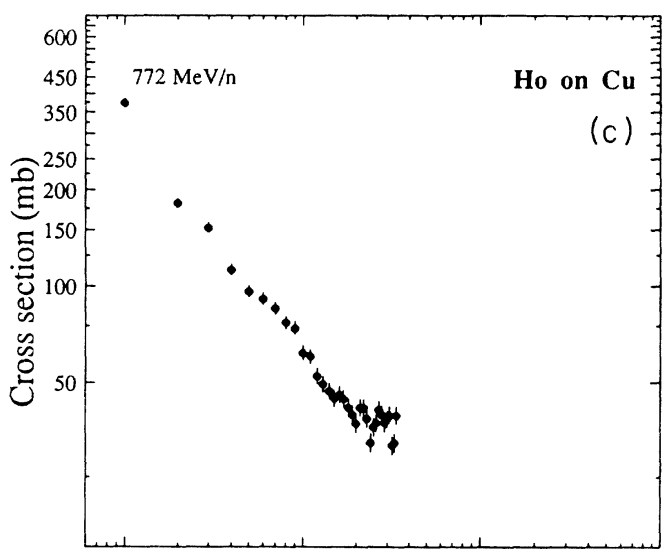

Charge change $(\Delta \mathrm{Z})$

FIG. 9. (a)-(c) Partial cross sections, in mb, for Ho at several energies on $\mathrm{C}, \mathrm{Al}$, and $\mathrm{Cu}$ as a function of $\Delta Z$. Plotted on log-log scales, each energy run is displaced by a multiplicative factor from the preceding run for clarity, with the highest-energy run further displaced to the right. The individual energies are listed in Table II.

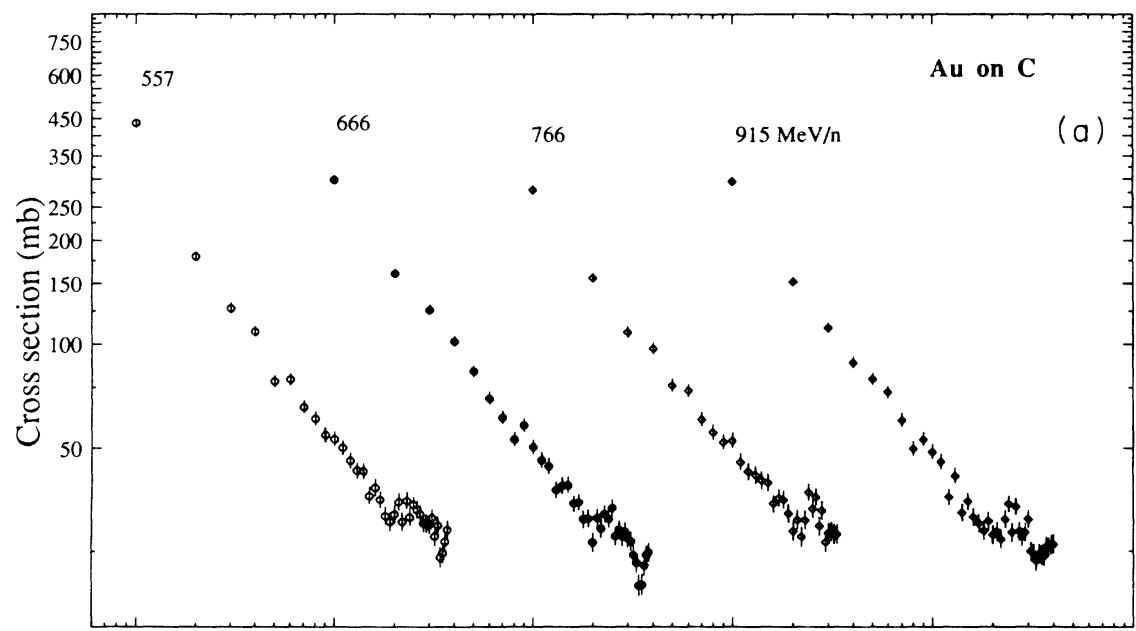

Charge change $(\Delta \mathrm{Z})$

FIG. 10. (a)-(c) Partial cross sections, in $\mathrm{mb}$, for $\mathrm{Au}$ at four energies on $\mathrm{C}, \mathrm{Al}$, and $\mathrm{Cu}$ as a function of $\Delta Z$. Plotted on log-log scales, each energy run is displaced by a multiplicative factor from the preceding run for clarity, with the highest-energy run further displaced to the right. The individual energies are listed in Table II. 


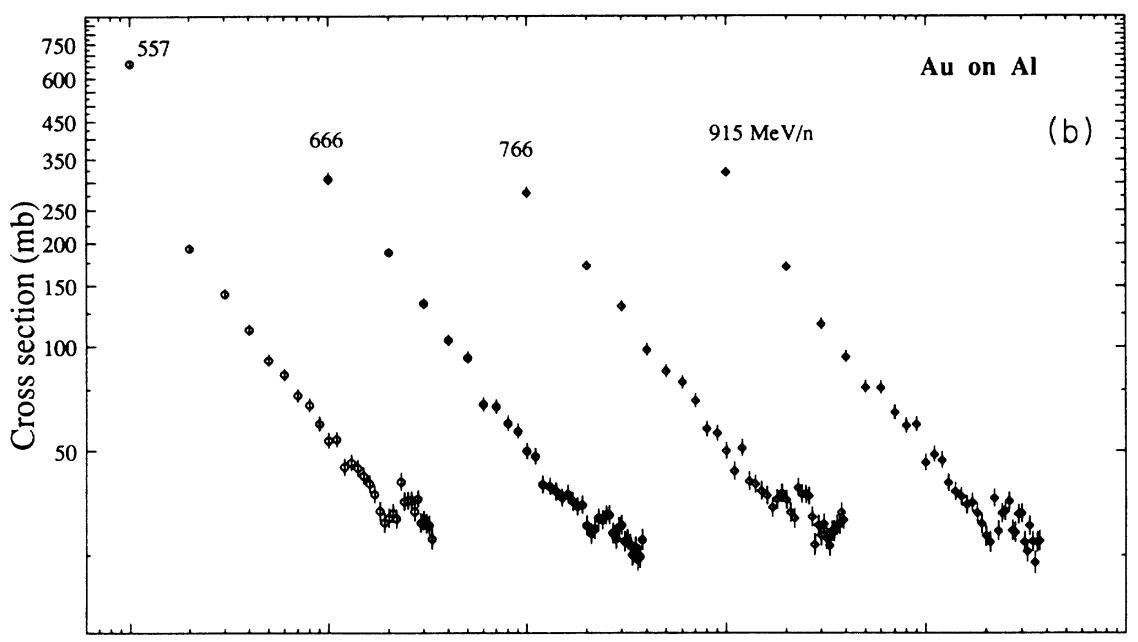

Charge change $(\Delta \mathrm{Z})$

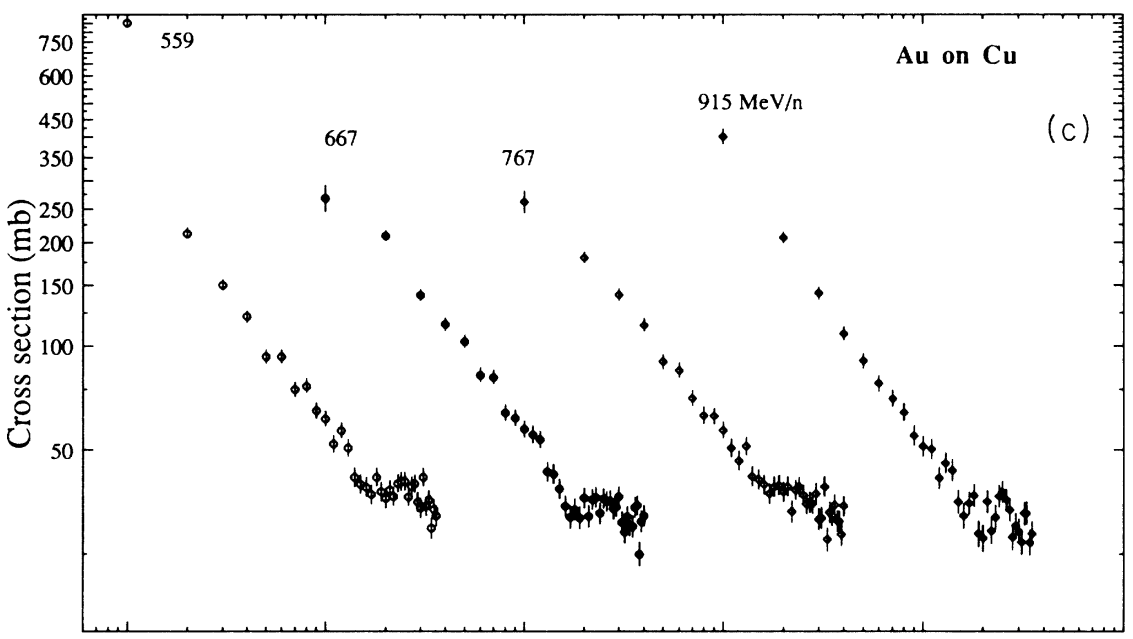

Charge change $(\Delta \mathrm{Z})$

FIG. 10. (Continued).

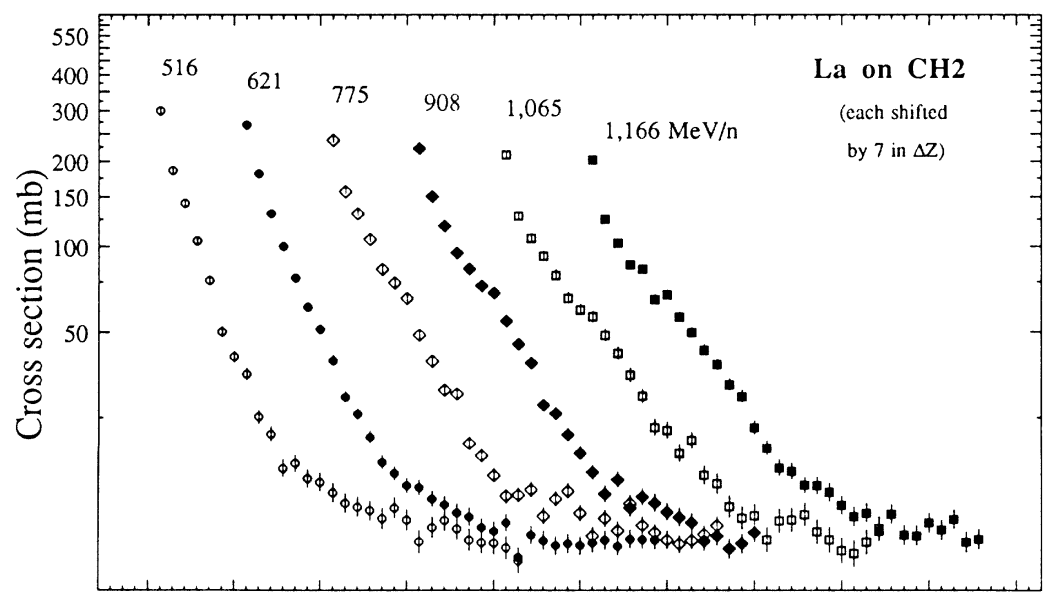

Charge change $(\Delta \mathrm{Z})$

FIG. 11. Partial cross sections, in mb, for $\mathrm{La}$ at six different energies on $\mathrm{CH}_{2}$ as a function of $\Delta Z$. Plotted on log-linear scale, each energy run is displaced by an additive factor from the preceding run for clarity, with the highest-energy run furthest displaced to the right. The individual energies are listed in Table II. 


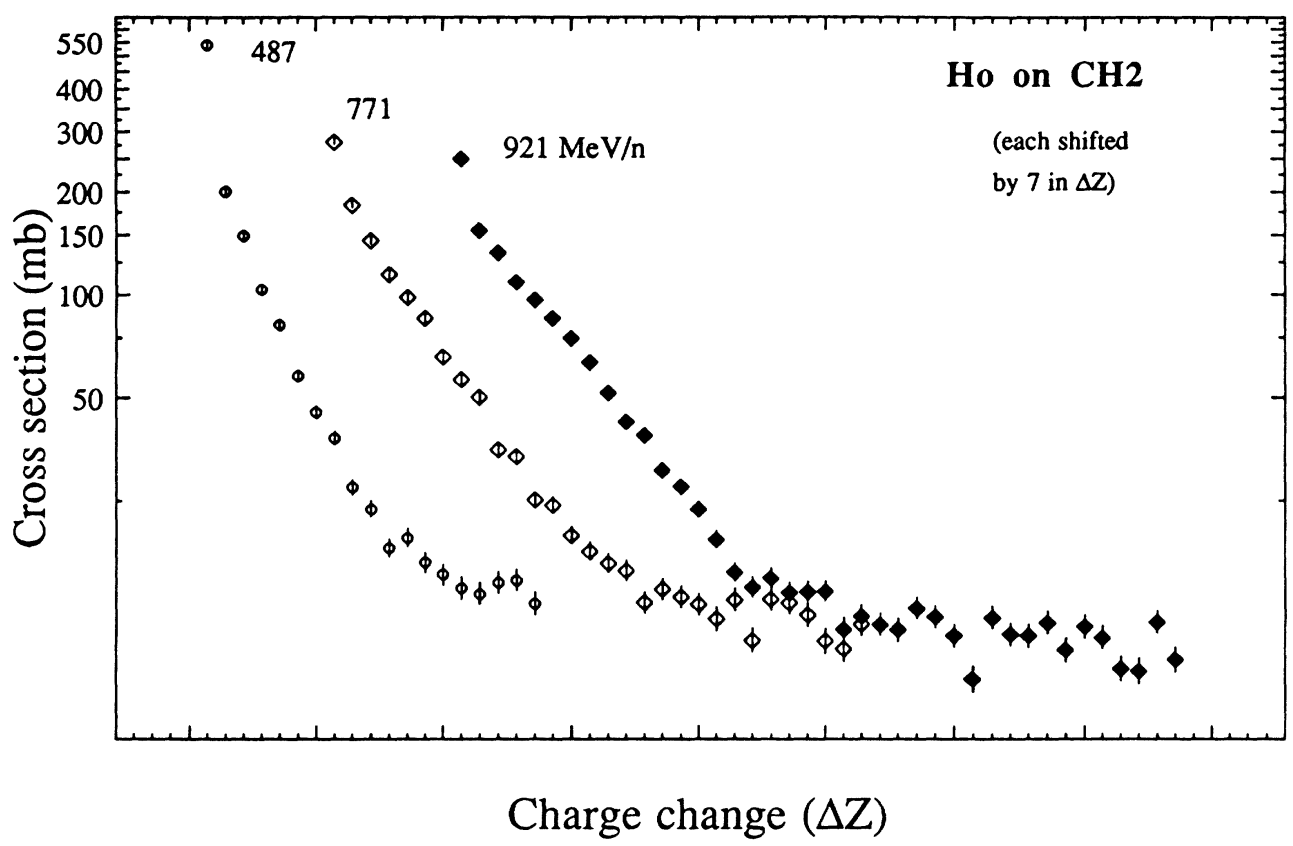

FIG. 12. Partial cross sections, in mb, for $\mathrm{Ho}$ at three different energies on $\mathrm{CH}_{2}$ as a function of $\Delta Z$. Plotted on log-linear scale, each energy run is displaced by an additive factor from the preceding run for clarity, with the highest-energy run further displaced to the right. The individual energies are listed in Table II.

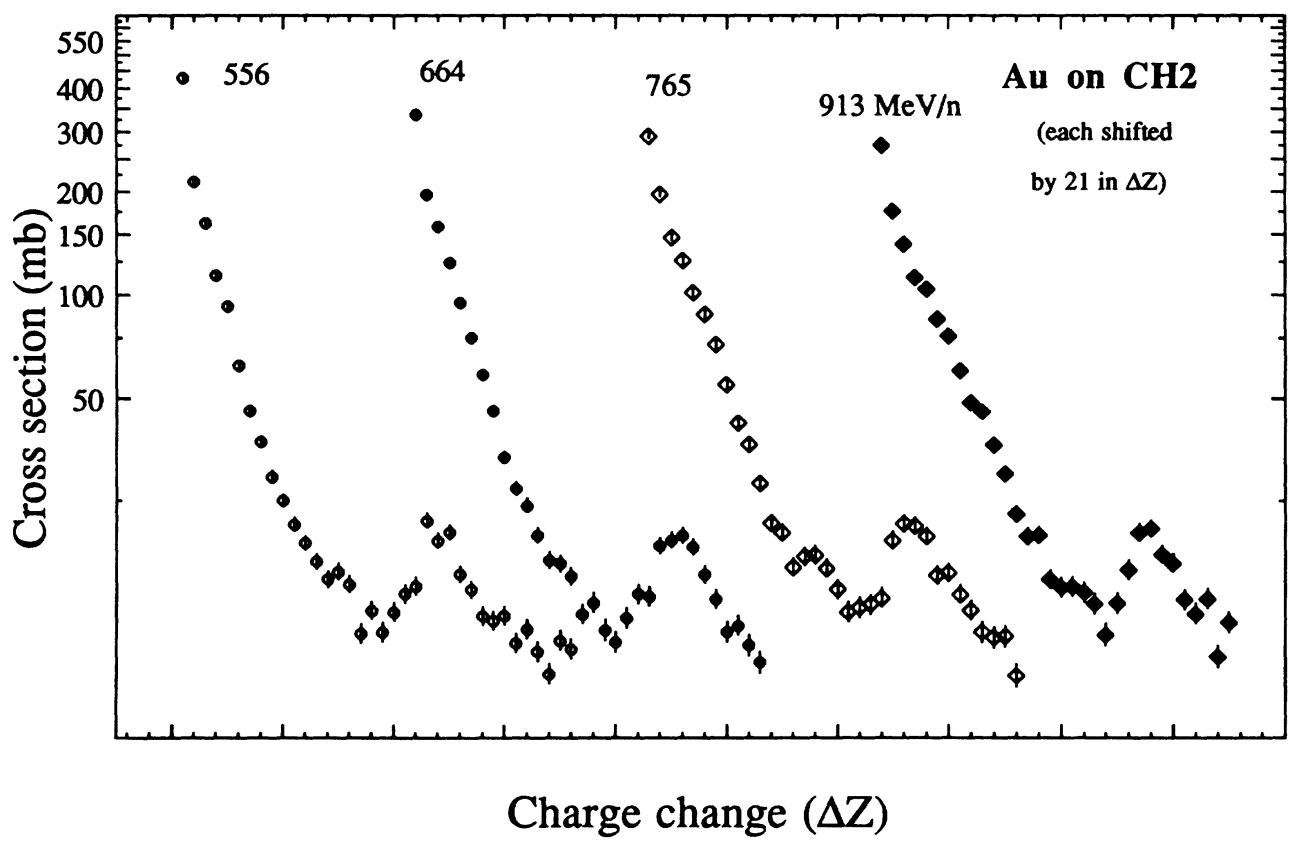

FIG. 13. Partial cross sections, in mb, for $\mathrm{Au}$ at four different energies on $\mathrm{CH}_{2}$ as a function of $\Delta Z$. Plotted on log-linear scale, each energy run is displaced by an additive factor from the preceding run for clarity, with the highest-energy run further displaced to the right. The individual energies are listed in Table II. 


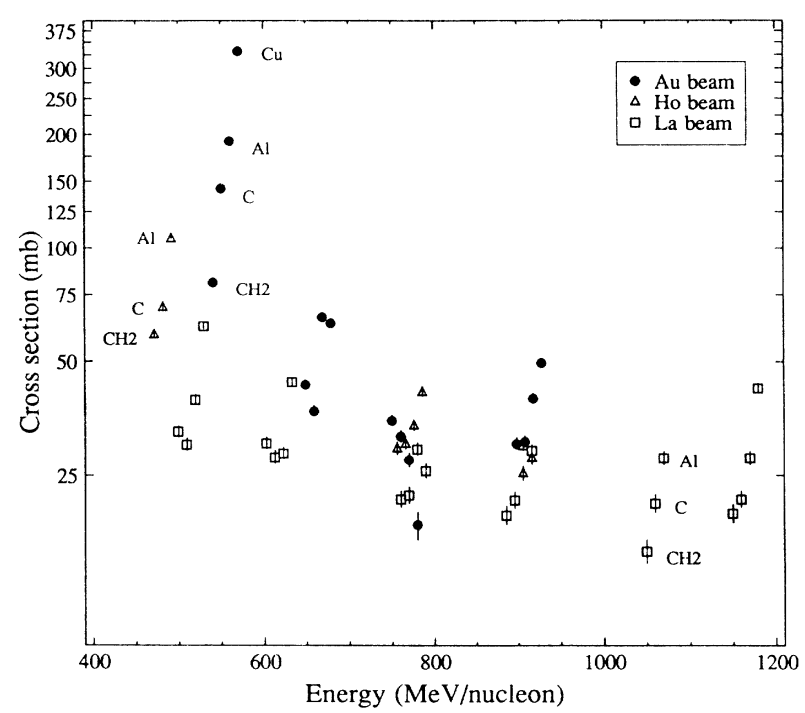

FIG. 14. Partial cross sections, in $\mathrm{mb}$, for $\Delta Z=+1$ as a function of energy. For clarity, runs made at a similar energy have been displaced in energy, with the lighter targets to lower and the heavier targets to higher energies. Some individual points are labeled with the target type.

and are available on request. ${ }^{25}$ The cross sections cover a range of values of $\Delta Z=+1$ to -36 . The uncertainties for the cross sections reprinted from Kertzman's thesis ${ }^{14}$ have been modified to include statistical uncertainties from the thick-target correction, and hence are a very small amount $(\approx 5 \%)$ larger than reported here. In addition, for the $\mathrm{CH}_{2}$ targets only, an uncertainty in the thick-target correction program caused the reported cross sections for $|\Delta Z| \geq 10$ to be a few percent too large. This has been corrected.

We did not use a polyethylene target with an iron projectile, so we could not deduce the cross sections for iron on a hydrogen target. Webber et al. ${ }^{26}$ have published data for iron on hydrogen cross sections and also for iron other targets. The agreement between our results is good.

\section{Cross sections for positive charge change}

Results on the production of elements with $\Delta Z=+1$ have been reported by us previously ${ }^{27}$ for some of the runs discussed here. We found that for heavy-projectile nuclei, cross sections for these charge pick-up fragments were about $10 \%$ of the cross sections for production of fragments with $\Delta Z=-1$. We have now found that an iron projectile also can undergo a positive charge change, but with cross sections that are relatively much smaller, being only about $1 \%$ of the $\Delta Z=-1$ cross section. We have also measured these cross sections at more energies and for Ho projectile nuclei. All our available values are listed in Table $\mathrm{V}$, and, with the exception of those for $\mathrm{Fe}$, are shown in Fig. 14. It can be seen that at all energies these cross sections are dependent on the mass of the target nucleus, generally increasing as the mass increases. The energy dependencies can be more clearly appreciated by scaling these cross sections with the total interaction
TABLE V. Cross sections for $\Delta Z=+1$ fragments.

\begin{tabular}{|c|c|c|c|}
\hline Beam & Target & $\begin{array}{c}\text { Energy } \\
(\mathrm{MeV} / \text { nucleon })\end{array}$ & $\begin{array}{c}\text { Cross section } \\
\text { for } \Delta Z=+1 \\
\quad(\mathrm{mb})\end{array}$ \\
\hline $\mathrm{Fe}$ & $\mathrm{C}$ & 1569 & $0.63 \pm 0.25$ \\
\hline $\mathrm{Fe}$ & $\mathrm{Al}$ & 1569 & $0.98 \pm 0.40$ \\
\hline $\mathrm{Fe}$ & $\mathrm{Cu}$ & 1569 & $1.18 \pm 0.66$ \\
\hline $\mathrm{Fe}$ & $\mathrm{Pb}$ & 1563 & $2.07 \pm 1.30$ \\
\hline $\mathrm{La}$ & $\mathbf{H}$ & 516 & $33.9 \pm 3.3$ \\
\hline $\mathrm{La}$ & $\mathbf{H}$ & 621 & $31.9 \pm 2.5$ \\
\hline $\mathrm{La}$ & $\mathbf{H}$ & 776 & $21.5 \pm 2.1$ \\
\hline $\mathrm{La}$ & $\mathbf{H}$ & 906 & $18.8 \pm 2.1$ \\
\hline $\mathbf{L a}$ & $\mathbf{H}$ & 1065 & $13.2 \pm 2.6$ \\
\hline $\mathrm{La}$ & $\mathbf{H}$ & 1166 & $19.0 \pm 2.3$ \\
\hline $\mathrm{La}$ & $\mathrm{C}$ & 516 & $30.3 \pm 3.1$ \\
\hline $\mathrm{La}$ & $\mathrm{C}$ & 621 & $28.0 \pm 2.1$ \\
\hline $\mathrm{La}$ & $\mathrm{C}$ & 777 & $22.3 \pm 1.8$ \\
\hline $\mathrm{La}$ & $\mathrm{C}$ & 903 & $21.6 \pm 1.8$ \\
\hline $\mathrm{La}$ & $\mathrm{C}$ & 1065 & $21.2 \pm 2.2$ \\
\hline $\mathrm{La}$ & $\mathrm{C}$ & 1166 & $21.7 \pm 1.8$ \\
\hline $\mathrm{La}$ & $\mathrm{Al}$ & 515 & $39.6 \pm 5.3$ \\
\hline $\mathrm{La}$ & $\mathrm{Al}$ & 618 & $28.7 \pm 2.4$ \\
\hline $\mathrm{La}$ & Al & 775 & $29.4 \pm 2.4$ \\
\hline $\mathrm{La}$ & $\mathrm{Al}$ & 901 & $30.2 \pm 2.2$ \\
\hline $\mathrm{La}$ & $\mathrm{Al}$ & 1080 & $27.9 \pm 3.2$ \\
\hline $\mathrm{La}$ & $\mathrm{Al}$ & 1165 & $27.9 \pm 2.4$ \\
\hline $\mathrm{La}$ & $\mathrm{Cu}$ & 515 & $62.1 \pm 10.5$ \\
\hline $\mathrm{La}$ & $\mathrm{Cu}$ & 618 & $44.1 \pm 3.8$ \\
\hline $\mathrm{La}$ & $\mathrm{Cu}$ & 775 & $25.8 \pm 3.1$ \\
\hline $\mathrm{La}$ & $\mathrm{Cu}$ & 901 & $29.2 \pm 2.6$ \\
\hline $\mathrm{La}$ & $\mathrm{Cu}$ & 1165 & $42.6 \pm 4.2$ \\
\hline Ho & $\mathbf{H}$ & 488 & $53.7 \pm 4.4$ \\
\hline Ho & $\mathbf{H}$ & 772 & $29.2 \pm 2.7$ \\
\hline Ho & $\mathbf{H}$ & 992 & $24.1 \pm 2.4$ \\
\hline Ho & $\mathrm{C}$ & 489 & $69.5 \pm 3.8$ \\
\hline Ho & $\mathrm{C}$ & 772 & $30.5 \pm 2.1$ \\
\hline Ho & $\mathrm{C}$ & 922 & $27.9 \pm 2.2$ \\
\hline Ho & $\mathrm{Al}$ & 489 & $106.3 \pm 6.5$ \\
\hline Ho & $\mathrm{Al}$ & 772 & $33.9 \pm 2.6$ \\
\hline Ho & $\mathrm{Cu}$ & 772 & $41.5 \pm 4.0$ \\
\hline $\mathrm{Au}$ & $\mathrm{H}$ & 557 & $49.6 \pm 4.3$ \\
\hline $\mathrm{Au}$ & $\mathrm{H}$ & 665 & $46.6 \pm 3.2$ \\
\hline $\mathrm{Au}$ & $\mathbf{H}$ & 766 & $36.6 \pm 2.9$ \\
\hline $\mathrm{Au}$ & $\mathrm{H}$ & 914 & $30.3 \pm 2.6$ \\
\hline $\mathrm{Au}$ & $\mathrm{C}$ & 557 & $144.3 \pm 4.9$ \\
\hline $\mathrm{Au}$ & $\mathrm{C}$ & 666 & $37.1 \pm 2.9$ \\
\hline $\mathrm{Au}$ & $\mathrm{C}$ & 766 & $31.9 \pm 2.6$ \\
\hline $\mathrm{Au}$ & $\mathrm{C}$ & 915 & $30.9 \pm 2.2$ \\
\hline $\mathrm{Au}$ & $\mathrm{Al}$ & 557 & $192.4 \pm 6.8$ \\
\hline $\mathrm{Au}$ & $\mathrm{Al}$ & 666 & $65.8 \pm 5.2$ \\
\hline $\mathrm{Au}$ & $\mathrm{Al}$ & 766 & $27.6 \pm 3.9$ \\
\hline $\mathrm{Au}$ & $\mathrm{Al}$ & 915 & $40.1 \pm 3.1$ \\
\hline $\mathrm{Au}$ & $\mathrm{Cu}$ & 559 & $333.7 \pm 12.2$ \\
\hline $\mathrm{Au}$ & $\mathrm{Cu}$ & 667 & $63.2 \pm 9.0$ \\
\hline $\mathrm{Au}$ & $\mathrm{Cu}$ & 767 & $18.7 \pm 7.3$ \\
\hline $\mathrm{Au}$ & $\mathrm{Cu}$ & 915 & $49.8 \pm 6.0$ \\
\hline
\end{tabular}



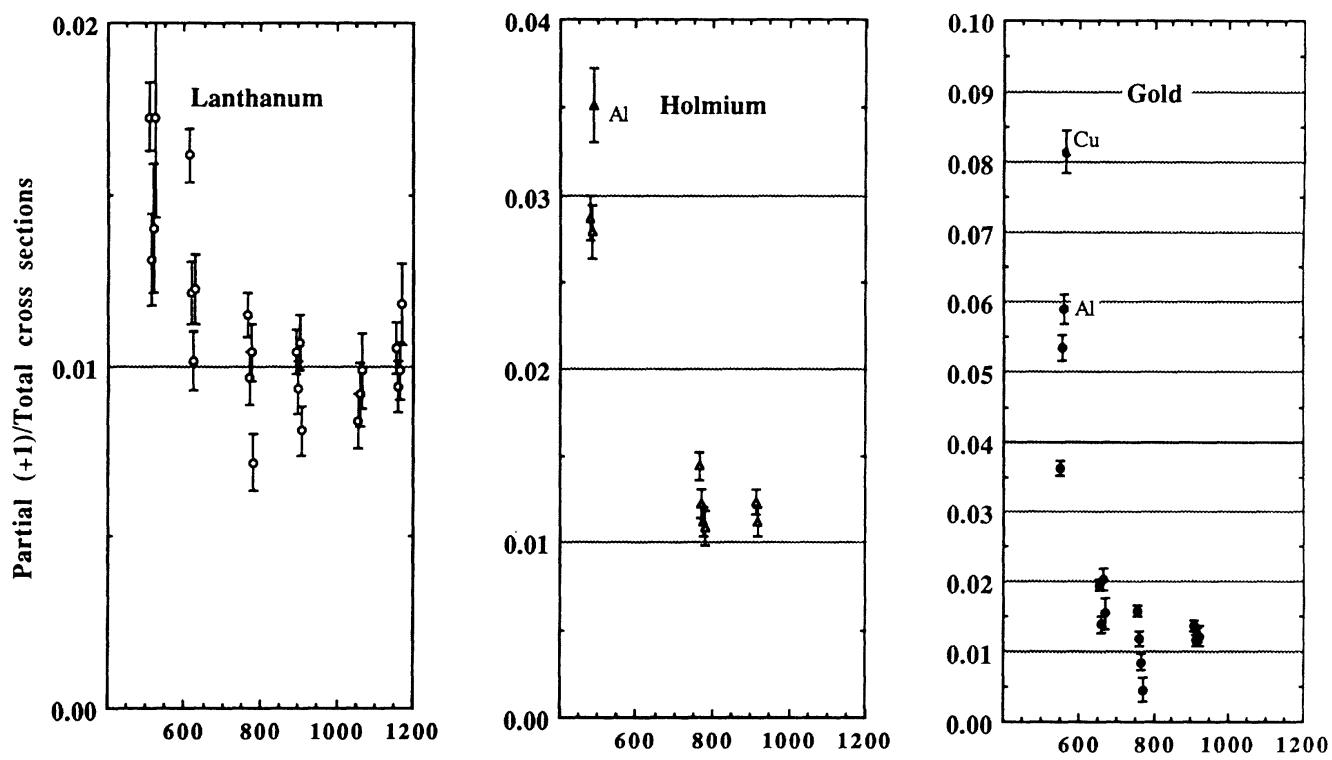

Energy (MeV/nucleon)

FIG. 15. Scaled partial cross sections $\sigma / \Sigma$ for $\Delta Z=+1$ as a function of energy for each of the three main projectiles. For clarity runs made at a similar energy have been displaced in energy, with the lighter targets to lower and the heavier targets to higher energies. Note the progressive change in vertical scales with increasing projectile mass. Some individual points are labeled with the target type.

cross sections $\Sigma$ from Eq. (1), as shown in Fig. 15. It can be seen that above $\approx 700 \mathrm{MeV} /$ nucleon these scaled cross sections are relatively independent of energy and projectile, with $\sigma(\Delta Z=+1) \approx 1 \%$ of $\Sigma$. However, there are strong dependences below $700 \mathrm{MeV} /$ nucleon on both projectile and target. While La has only a slight energy dependence, Ho shows a significant energy dependence, but one that is relatively independent of the target, although there was no available $\mathrm{Cu}$ target data at the lowest energy. Au has a large energy dependence that is strongly target dependent, with $\mathrm{Au}$ on $\mathrm{Cu}$ at 557 $\mathrm{MeV} /$ nucleon having $\sigma(\Delta Z=+1)$ as great as $8 \%$ of $\Sigma$. At intermediate energies, $\mathrm{Au}$ at $766 \mathrm{MeV} /$ nucleon, the target dependence appears to reverse, an indication of the complexity of the process. It clearly would be of great interest to study these cross sections at still lower energies, but unfortunately this detector is not suited to such measurements.

A few isolated events due to the apparent production of fragments with $\Delta Z=+2$ have been observed in some of the runs. The number of these events is so small that at best we can place upper limits on the cross sections of the order of $1 \%$ of the cross section for $\Delta Z=+1$. However, enough events were seen to exclude the possibility that they were merely the consequence of two successive interactions. Within the statistics these results are consistent with the report by Guoxiao et al. ${ }^{28}$ of detecting two such events with $88 \Delta Z=+1$ events from a La projectile of $1.28 \mathrm{GeV} /$ nucleon in a CR-39 target. These observations in plastic, where the charge change is observed to occur between two sheets, prove that these $\Delta Z=+2$ fragments are not formed by successive $\Delta Z=+1$ interactions, nor by decay of an excited fragment. An order of magnitude greater statistics would be needed to establish reasonable estimates of the true cross sections for these events.

\section{E. Determination of some total charge-changing cross sections}

Because of the triggering conditions used in this experiment we could only determine the total charge-changing

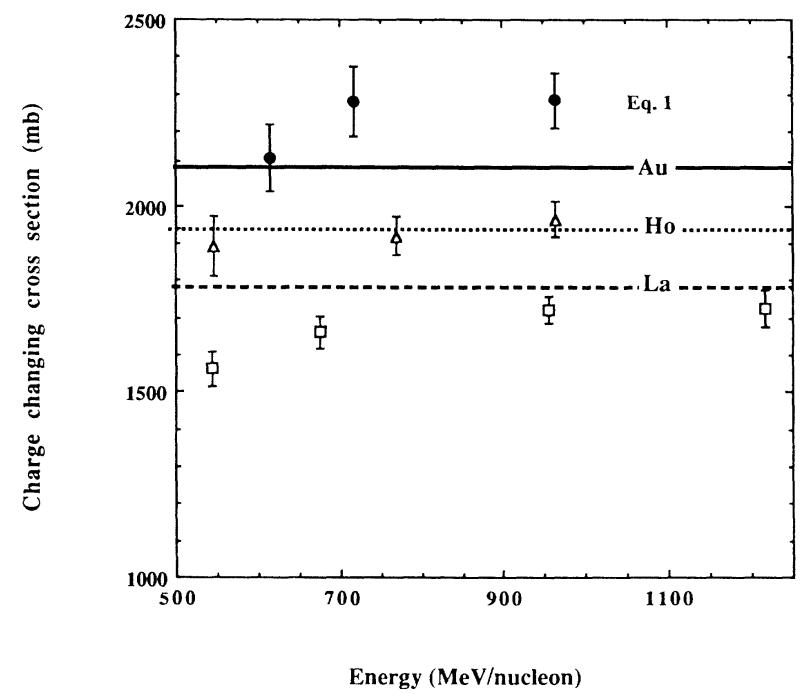

FIG. 16. Total charge-changing cross sections $\Sigma$, in $\mathrm{mb}$, in the material of the Cherenkov detectors, as a function of energy, compared with the predictions of Eq. (1), shown as horizontal lines. 
TABLE VI. Total charge-changing cross sections in the Cherenkov counter material, compared with the predictions from Eq. (1). The approximate $1 \%$ uncertainty on the value from Eq. (1) is estimated from the quoted deviation from the data to which it was fitted (Ref. 8).

\begin{tabular}{lcccc}
\hline \hline Beam & $\begin{array}{c}\text { Energy } \\
(\mathrm{MeV} / \text { nucleon) }\end{array}$ & $\begin{array}{c}\text { Number } \\
\text { in run }\end{array}$ & $\begin{array}{c}\text { Number } \\
\text { surviving } \\
\text { (from fit) }\end{array}$ & $\begin{array}{c}\text { Total charge-changing } \\
\text { cross section (mb) } \\
\text { per target nucleus }\end{array}$ \\
\hline $\mathrm{La}$ & 543 & 164901 & 133493 & $1562 \pm 48$ \\
$\mathrm{La}$ & 675 & 165074 & 131849 & $1661 \pm 42$ \\
$\mathrm{La}$ & 956 & 165074 & 130789 & $1721 \pm 37$ \\
$\mathrm{La}$ & 1216 & 90961 & 72034 & $1724 \pm 50$ \\
$\mathrm{La}$ & Eq. (1) & & & $1785 \pm 18$ \\
$\mathrm{Ho}$ & 547 & 87012 & 67371 & $1891 \pm 82$ \\
$\mathrm{Ho}$ & 769 & 165074 & 127301 & $1920 \pm 52$ \\
$\mathrm{Ho}$ & 962 & 165074 & 126555 & $1964 \pm 46$ \\
$\mathrm{Ho}$ & Eq. (1) & & & $2129 \pm 88$ \\
$\mathrm{Au}$ & 614 & 165074 & 123759 & $2280 \pm 95$ \\
$\mathrm{Au}$ & 718 & 158665 & 116545 & $2283 \pm 74$ \\
$\mathrm{Au}$ & 964 & 164886 & 121067 & $2121 \pm 21$ \\
$\mathrm{Au}$ & Eq. (1) & & & \\
\hline \hline
\end{tabular}

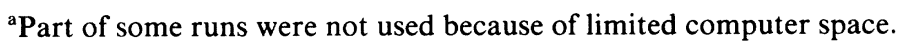

cross sections for the case of particles interacting in the material of the Cherenkov counter. In this case, by using the ion chambers on either side of the Cherenkov counter, we could measure the number of projectile particles that enter and compare the number with the number that survive. For several target-out runs at various energies, we considered only those events which passed all the selections except for particles interacting in the material of the detector. It was then required that the events be projectile particles and have signals in the second ion counter (I-2 following the location of the target holder) within 2 standard deviations of the peak signal of the projectile. This data set selected only particles which were projectile particles as they entered the front of the Cherenkov detector. Emerging particles were analyzed by fitting Gaussian peaks to a histogram of the sum of the signals of the last ion counters (I-3 and I-4) in the region of the histogram near the projectile peak. The number of surviving non-charge-changed particles in the projectile peak compared to the total number of incident particles, together with the knowledge of the thickness of the materials, gave the total charge-changing cross section for the mixture of materials in the Cherenkov detector. The residual uncertainties due to the nonresolved $\Delta Z=-1$ fragments can hardly exceed $10 \%$, due to the size of the partial cross section, and the correction made for these fragments should be good at least $10 \%$; so any residual uncertainties due to this effect must be less than $1 \%$.

The cross sections determined in this way are shown as a function of energy for each projectile in Fig. 16, and are listed in Table VI. In both cases they are compared with the energy-independent cross sections found from Eq. (1), taking into account all the differing materials in the Cherenkov counters, Table I. The data suggest that there is apparently a small energy dependence of the total cross sections, with the cross sections increasing as the energy increases. There is also an indication that cross sections increase faster with projectile mass than indicated by Eq. (1). However, given the insensitivity of the determination of the partial cross-sections to the assumed value of the total cross section, where a $10 \%$ uncertainty in the total cross sections results in a $1 \%$ uncertainty in the partial cross sections, it is acceptable to neglect second-order variations in this analysis. Hence we used Eq. (1) for the total charge-changing cross sections of heavy nuclei throughout. Additional measurements of the total cross sections are needed to refine Eq. (1) for these heavy projectiles, particularly on targets that contain hydrogen.

\section{MASS LOSSES IN INTERACTIONS}

\section{A. Introduction}

Nuclear interactions will normally result in changes in the neutron number $(\Delta N)$ as well as in the proton number $\Delta Z$. There is also a non-negligible cross section for interactions with finite $\Delta N$ but zero $\Delta Z$. A knowledge of the cross sections for production of all the isotopes is important for application to the problem of cosmic ray propagation and to understanding the nuclear physics that is occurring. However, at the high energies of interest to comic ray physicists, isotopic identification is difficult, and in this range of masses, beyond current technology.

The semiempirical cross-section formulas ${ }^{29}$ predict larger than proportional neutron losses in small charge loss interactions and also large neutron losses for charge pick-up interactions. Our results from the 1986 experiment confirm these qualitative statements but differ significantly quantitatively. We have analyzed these small $\Delta Z$ interactions in the lower energy $\mathrm{La}$ and $\mathrm{Au}$ runs in a previous paper, ${ }^{27}$ and the reader is referred 
there for the experimental details. Here we will concentrate on the mass losses observed in interactions with larger values of $\Delta Z$.

\section{B. Method of mass discrimination}

The detector was not designed to detect isotopes, but the presence of material in the detector allows a degree of mass discrimination. The signals in the detector are dependent both on the charge and velocity of the nuclei. The velocities of the nuclei decrease as they pass through the detector due to ionization energy losses. The magnitude of the total energy loss depends on the charge but not the mass of the nuclei, but the velocity change also depends on the mass, being greater for lighter isotopes than for heavier isotopes of the same element produced in fragmentation. Thus the projectile nuclei have a welldefined velocity throughout the detector, but fragments have velocities that may be greater or lesser than that of the projectile according to whether the energy loss per nucleon of the fragments is less, or greater, than that of the projectile. Thus a histogram of the velocities of fragments of a given charge is composed from a series of peaks with the heavy isotopes at the high-velocity end and the light isotopes at the low-velocity end.

The Cherenkov detector provides a signal for each particle which is strongly dependent on the velocity. Once the charge of a particle is determined, the Cherenkov signal can be converted to a velocity. However, there are contributions to an energy spread among the fragment nuclei besides the differential energy per nucleon loss of different isotopes. Most notably, since the targets have a finite thickness, one fragment may have interacted near the front of the target while another with the same mass and charge may have been produced near the back of the target. Thus in the former case, the nucleus will have lost energy at a rate proportional to $\left(Z_{P}+\Delta Z\right)^{2}$ through the target, while in the latter case, the nucleus will have lost energy at the same rate as the projectile nuclei, proportional to $Z_{P}^{2}$, throughout the target. In addition, the original projectile will have a finite range of velocities. Finally, the instrumental resolution at a fixed velocity and charge is limited by photon statistical fluctuations.

The greatest rate of energy loss, and so the largest differential energy loss, occurs at the lower energies of the energy range available to us from the Belavac, but even in these cases actual resolution of isotopic peaks is not possible. However, it is possible to find an average energy loss of the fragments of a given charge, and so approximate average mass loss. In addition, starting with an assumed set of cross sections for the production of each isotope we can generate an expected distribution of velocities, by folding in the information about energy loss for a given charge and mass, the target thickness, and the instrumental resolution. Thus, with an assumption about the shape of the isotopic distribution of fragments, we can fit a distribution to the Cherenkov signal-velocity histogram.

\section{Fits to the Cherenkov signal data}

The simplest distribution for the isotopic composition of the fragments of a given charge which is consistent with the available information is a Gaussian one. We have fitted the data with Gaussian distributions of isotopes and obtained good values of reduced chi squared, $\chi^{2}$. The fits are not as good for an assumed square distribution of isotopes. However, the fit would be just as good for Gaussian distributions with every other isotope completely missing. Hence the fits are really only useful for determining mean mass losses, rather than the detailed shape of the isotopic distribution. These fits would also be distorted if there were appreciable numbers of other light fragments or meson production affecting the shape of the distributions. Such effects would cause us to underestimate the true mass losses.

A Gaussian distribution has also been used by Webber et al. ${ }^{26}$ to fit the isotopic distribution of fragments from lighter projectiles $(Z<28)$. However, their fits indicate isotopic compositions much closer to the line of stability with respect to beta decay than do our results. Tentatively, we may account for this by the fact that there is less binding energy per nucleon for the heavier nuclei we are using and the lighter fragments have narrower valleys of stability.

The median mass losses obtained from such fits to the signals for two gold and one lanthanum run on a carbon target at low energy are shown on Fig. 17 for $|\Delta Z| \leq 13$, compared with the predictions of the semiempirical expressions $^{29}$ for a hydrogen target for $|\Delta Z| \leq 10$. The individual values are given elsewhere. ${ }^{24}$ It can be seen from the figure that there is a steady increase in the median mass loss as $|\Delta Z|$ increases, and that it is dependent on the mass of the projectile nucleus. It can also be seen that the predictions for a hydrogen target are not too different from the observations for a carbon target. This is in accord with the observation that for small $\Delta Z$ the median mass loss is essentially independent of the tar-

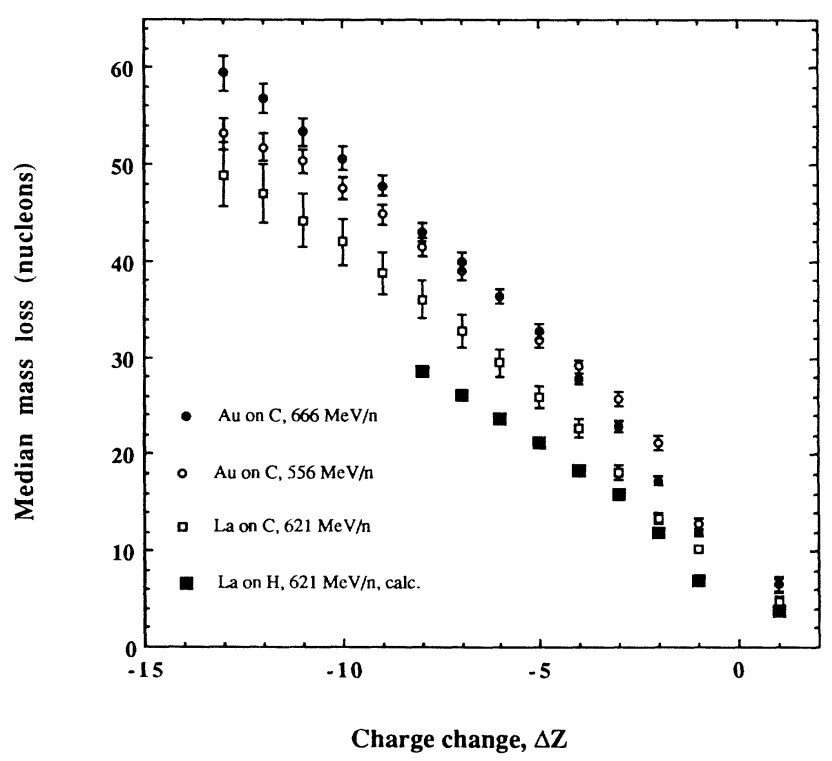

FIG. 17. Median mass changes, in nucleons, as a function of $\Delta Z$ for several runs on a carbon target. Also shown are the predictions of Silberberg, Tsao, and Letaw (Ref. 29) for La at 621 $\mathrm{MeV} /$ nucleon on a hydrogen target. 
get. ${ }^{27}$ These median mass losses can be scaled by $\Delta Z$ to give the median neutron loss per proton. These are shown in Fig. 18, where it can be seen that the median number of neutrons lost for each proton steadily decreases as $|\Delta Z|$ increases, as it clearly must do physically. The large neutron losses found indicate that for $|\Delta Z|$ as large as $10-15$, the fragments formed are very proton rich, and will decay in time to stable isotopes with $\Delta Z$ as much as 5-8 smaller than that of the initially produced fragments. Since these mass losses also have a wide range of values the composition of a decayed sample of fragments will be significantly different from the sample observed directly after the interactions, and thus affect any calculation of cosmic ray propagation. It should be noted that none of these median losses are so large that they come close to predicting physically unreasonable nuclei with lifetimes less than those necessary for transit of the detector. (This analysis assumes that the fragment experiences no energy loss in the interaction. ${ }^{27}$ Introducing an energy loss will slightly reduce the calculated neutron loss. For example, a mean energy loss of $5 \mathrm{MeV} /$ nucleon when 621$\mathrm{MeV} /$ nucleon La produces a $\Delta Z=-13$ fragment only reduces the median mass loss from 49 to 45 nucleons.)

The uncertainties on our estimates of these losses are larger for the higher-energy runs than for the lowerenergy runs, because the instrumental resolution becomes more important relative to the signal difference caused by differential energy loss of isotopes. The uncertainties for the lowest-energy lanthanum runs are large because we used thinner targets in these runs and so had less differential energy loss than for thicker-target runs. At approximately $\Delta Z=-15$, the varied composition of the subsidiary fragments begins to play a role in the spread of Cherenkov signals for the major fragment. The subsidiary fragments may be protons, alpha particles, or larger

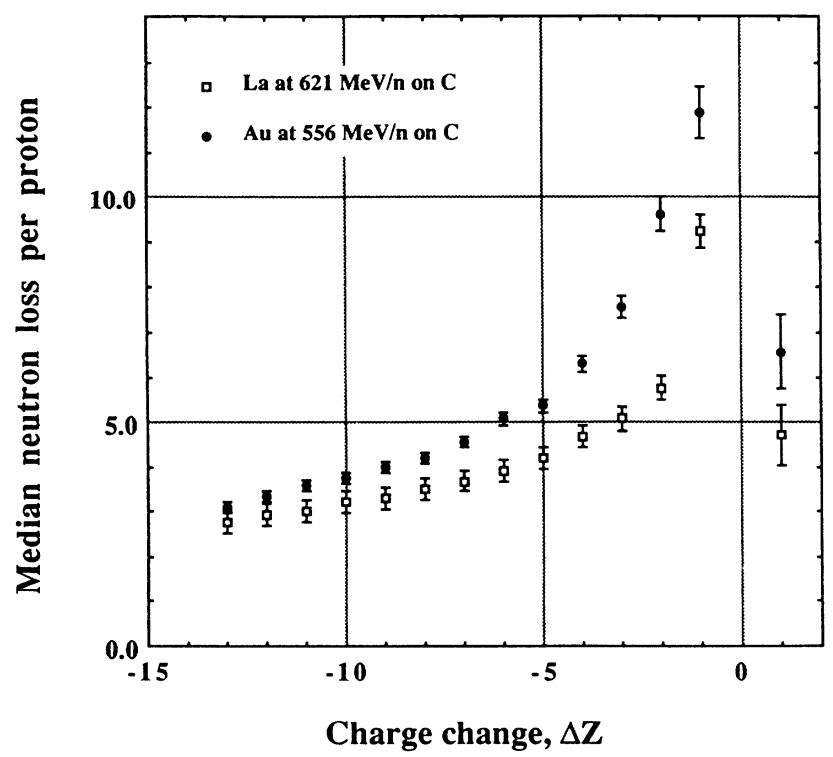

FIG. 18. Median neutron loss per proton as a function of $\Delta Z$ for a $\mathrm{La}$ and a $\mathrm{Au}$ run on a carbon target. For $\Delta Z=+1$ the values shown are for the medium mass loss. nuclei. The width of the mass distributions obtained from fitting the Cherenkov signals remains approximately constant down to about $\Delta Z=-10$. Then the width begins to increase with increasing $|\Delta Z|$ and by -15 has become significantly larger. For example, consider two interactions both occurring at the center of the target. Then for a projectile energy of $621 \mathrm{MeV} /$ nucleon, the signal of a fragment of a lanthanum nucleus which has lost 10 protons and 30 neutrons plus the signal of the 10 free protons would be equal to the signal of a fragment which has lost 10 protons and 31 neutrons plus the signal of $2 \alpha$ particles and 6 protons. Variable amounts of meson production would further confuse the analysis. Since we cannot at present predict the composition of the subsidiary fragments for a given interaction, we cannot with certainty find the mass distributions for large charge changes.

Studies of nuclear emulsion exposed to a 990 $\mathrm{MeV} /$ nucleon gold projectile $^{19}$ indicate that, in interactions in which gold nuclei break into large fragments plus subsidiary fragments, the subsidiary fragments are composed of approximately $72 \%$ singly charged particles and $24 \%$ doubly charged particles at a mean energy of about $600 \mathrm{MeV} /$ nucleon, and a decreasing percentage of multiply charged particles with increasing energy. ${ }^{30}$ Interactions may also result in the creation of pions. As collisions become more and more central they will lead to greater and greater charge changes, with a wider and wider spread in the number of pions being created, reducing the ability of detectors such as ours to determine the mean mass of the leading fragment. At much higher energies the production of large multiplicities of mesons must reduce the ability of this technique to measure even the charge of the fragment, since this depends on observing the total charge emitted from the interaction. For example, emulsion and counter measurements of 14.6, 60, and $200 \mathrm{GeV} /$ nucleon ${ }^{16} \mathrm{O}$ nuclei making interactions on heavy targets give mean meson multiplicities per wounded nucleon of $\approx 1.0, \approx 2.6$, and $\approx 4.0$, respectively. ${ }^{31-33}$ Even in peripheral collisions the number of wounded nucleons will increase rapidly with increasing $|\Delta Z|$, particularly with heavy targets, possibly enough that fluctuations in multiplicity from event to event will begin to obscure the resolution between charged fragments. The difference between the signals from a La projectile and a $\Delta Z=-1$ fragment is 113 singly charged particles, that for a $\mathrm{Au}$ projectile is 157 , but between $\Delta Z=-10$ and -11 fragments from $\mathrm{Au}$ it is 137 , or only $\approx 3.4$ particles per nucleon removed from the incident nucleus.

\section{CONCLUSIONS AND SUMMARY}

We have been able to study the production of fragments over a relatively wide range of $|\Delta Z|$ produced by heavy projectiles incident on a range of target nuclei with energies between 1.5 to 2.2 times the rest mass energy. The charge pick-up reactions with $\Delta Z=+1$ show cross sections with some very strong energy and target dependences, suggesting some form of resonance phenomena occurs for the lowest energies and heaviest targets. The 
charge-loss reactions with $\Delta Z \leq-1$ have cross sections that show significantly systematic trends. These systematics will be described in a companion paper and used to derive global fits to the data. The mean mass losses that we have studied show that from three to 12 neutrons are lost for each proton over the range of $\Delta Z$ that we can examine. Clearly this cannot continue out to much larger $\Delta Z$. Presumably, the point at which this rate of neutron loss decreases marks the transition from a peripheral interaction to a more central collision.

\section{ACKNOWLEDGMENTS}

We are grateful to all those who assisted in this experiment. In particular, we thank W. E. Althouse, J. A. Becker, J. W. Epstein, and B. W. Gauld of our own groups for their efforts. We are indebted to the staff of the LBL Bevalac facility, especially H. J. Crawford and J. M. Engelage for their essential assistance. This work was funded in part by NASA under Grant Nos. NAG 8-498, 8-500, and 8-502, and NGR 05-002-160, 24-005-050, and 26-008-001.
*Present address: California Institute of Technology, Pasadena, CA 91125.

1J. R. Cummings, T. L. Garrard, M. H. Israel, J. Klarmann, E. C. Stone, C. J. Waddington, and W. R. Binns, Phys. Rev. C 42, 2530 (1990), the following paper.

${ }^{2}$ W. R. Binns, T. L. Garrard, P. S. Gibner, M. H. Israel, M. P. Kertzman, J. Klarmann, B. J. Newport, E. C. Stone, and C. J. Waddington, Astrophys. J. 346, 997 (1989).

${ }^{3}$ See the review by J. Hufner, Phys. Rep. 125, 129 (1985).

${ }^{4}$ J. B. Cummings, P. E. Haustein, T. J. Ruth, and G. J. Virtes, Phys. Rev. C 17, 1632 (1978).

${ }^{5}$ D. J. Morrisey, W. R. Marsh, R. J. Otto, W. Loveland, and G. T. Seaborg, Phys. Rev. C 18, 1267 (1978).

${ }^{6}$ D. L. Olson, B. L. Berman, D. E. Greiner, H. H. Heckman, P. J. Lindstrom, and H. J. Crawford, Phys. Rev. C 28, 1602 (1983).

${ }^{7}$ N. T. Porile, G. D. Cole, and C. R. Rudy, Phys. Rev. C 19, 2288 (1979).

8J. Aichelin, A. Rosenhauer, G. Peilert, and H. Stocker, Proceedings of the 8th High-Energy Heavy-Ion Study, Report No. LBL-24580, 1988, p. 241.

${ }^{9}$ J. Randrup (Ref. 8), p. 246.

${ }^{10} \mathrm{C}$. Ngo, Proceedings of the 3rd International Conference on Nucleus-Nucleus Collisions, Saint Malo, 1988 [Nucl. Phys. A488, 133c (1988)].

${ }^{11}$ H. Ngo, C. Ngo, F. Ighezou, J. Desbois, S. Leray, and Zheng Yu Ming, Report No. LNS/PH/90-09, 1990, p. 105.

${ }^{12}$ N. R. Brewster, Ph.D. thesis, University of Minnesota, 1984; see also N. R. Brewster, R. K. Fickle, C. J. Waddington, W. R. Binns, M. H. Israel, M. D. Jones, J. Klarmann, T. L. Garrard, B. J. Newport, and E. C. Stone, in Proceedings of the 18th International Cosmic Ray Conference, Bangalore, 1983, edited by N. Durgaprasad et al. (Tata Institute of Fundamental Reseach, Bombay, 1983), Vol. 9, p. 259.

${ }^{13}$ W. R. Binns, T. L. Garrard, M. H. Israel, M. P. Kertzman, J. Klarmann, E. C. Stone, and C. J. Waddington, Phys. Rev. C 36, 1870 (1987).

${ }^{14}$ M. Kertzman, Ph.D. thesis, University of Minnesota, 1987.

${ }^{15}$ G. D. Westfall, L. W. Wilson, P. J. Lindstrom, H. J. Crawford, D. E. Greiner, and H. H. Heckman, Phys. Rev. C 19,
1309 (1979).

${ }^{16}$ H. C. Bradt and B. Peters, Phys. Rev. 77, 54 (1950).

${ }^{17}$ See the review by H. Boggild and T. Ferbel, Annu. Rev. Nucl. Sci. 24, 451 (1974).

${ }^{18}$ J. Klarmann, V. Vylet, C. J. Waddington, W. R. Binns, T. L. Garrard, and M. H. Israel, in Proceedings of the 21st International Cosmic Ray Conference, Adelaide, 1983, edited by R. J. Protheroe (Graphics Services, Northfield, South Australia, 1990), Vol. 4, p. 434.

${ }^{19}$ C. J. Waddington and P. S. Freier, Phys. Rev. C 31, 888 (1985).

${ }^{20}$ E. M. Friedlander, R. W. Gimpel, H. H. Heckman, Y. J. Karant, B. Judek, and E. Ganssauge, Phys. Rev. Lett. 45, 1084 (1981).

${ }^{21}$ H. B. Barber, P. S. Freier, and C. J. Waddington, Phys. Rev. Lett. 48, 856 (1982).

${ }^{22}$ E. M. Friedlander, H. H. Heckman, Y. J. Karant, and B. Judek, Phys. Rev. C 27, 1489 (1983).

${ }^{23}$ R. Bhanja et al., Phys. Rev. Lett. 54, 771 (1985).

${ }^{24}$ J. R. Cummings, Ph.D. thesis, University of Minnesota, 1989.

${ }^{25}$ Data available either printed or on Macintosh Excel spreadsheet from the University of Minnesota, or as part of the Transport Collaboration data base, LBL, H. Crawford.

${ }^{26}$ W. R. Webber, J. C. Kish, and D. A. Schrier, Phys. Rev. C 41, 520 (1990).

${ }^{27}$ W. R. Binns, J. R. Cummings, T. L. Garrard, M. H. Israel, J. Klarmann, E. C. Stone, and C. J. Waddington, Phys. Rev. C 39, 1785 (1989).

${ }^{28}$ R. Guoxiao, P. B. Price, and W. T. Williams, Phys. Rev. C 39, 1351 (1989).

${ }^{29}$ R. Silberberg, C. H. Tsao, and J. R. Letaw, Astrophys. J. Suppl. 58, 873 (1985); in Proceedings of the 20th International Cosmic Ray Conference, Moscow, 1987, edited by V. L. Kozyarivsky et al. (Nanka, Moscow, 1987), Vol. 2, p. 133.

${ }^{30}$ J. Achelin and X. Campi, Phys. Rev. C 34, 1643 (1986).

${ }^{31}$ KLM Collaboration, L. M. Barbier et al., Phys. Rev. Lett. 60, 405 (1988)

${ }^{32}$ EMU01 Collaboration, M. I. Admovich et al., Phys. Lett. B 227, 285 (1989). 


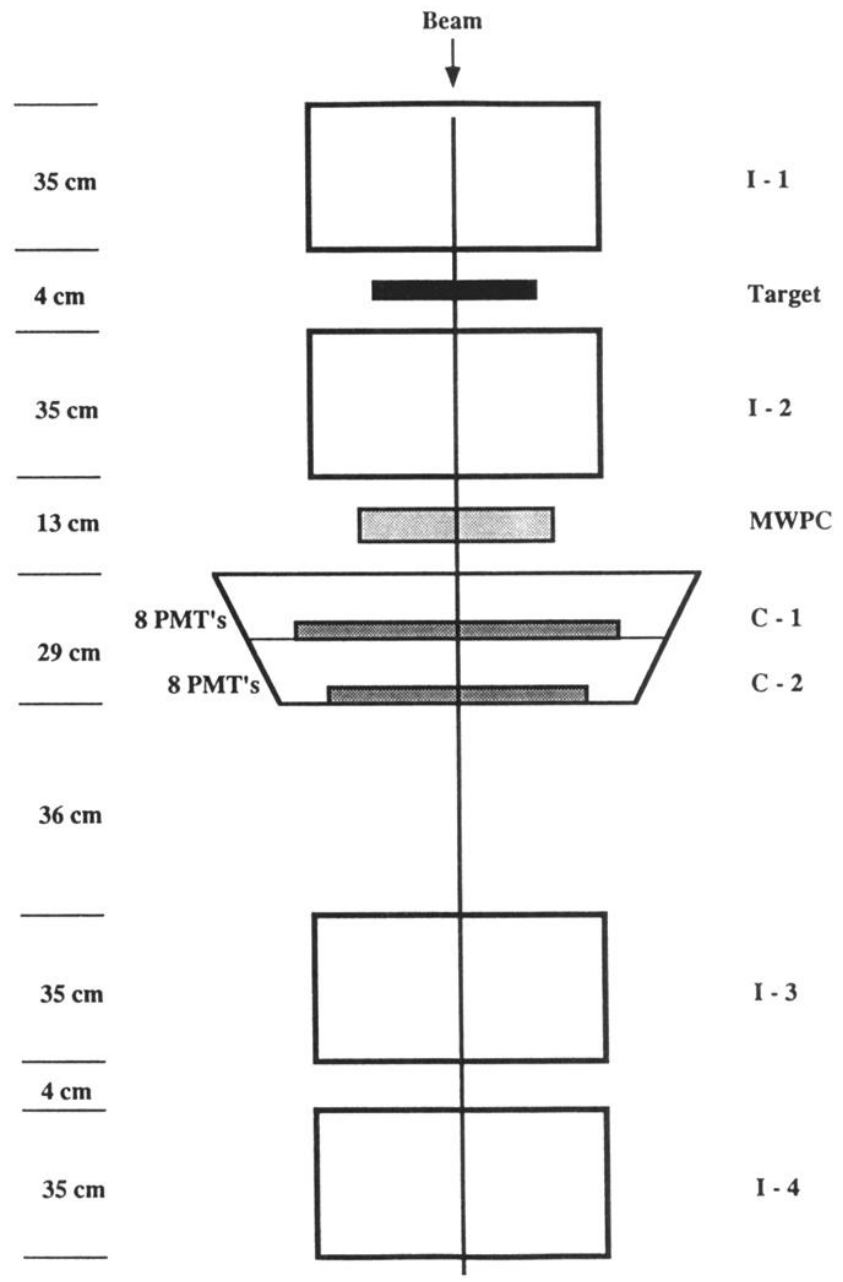

FIG. 1. Schematic diagram of the detector. Ionization counters are designated I and Cherenkov counters C. The target and the multiwire proportional counters, MWPC, are mounted as shown. 